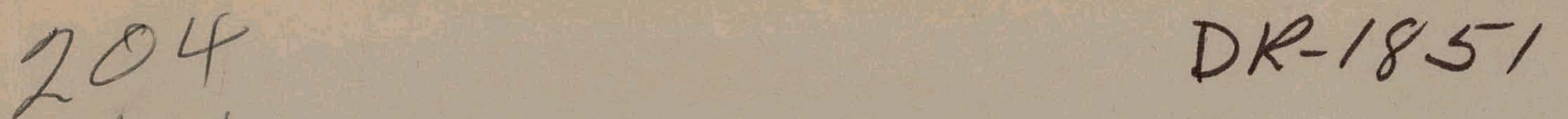

$1 / 7 / 71$

RFP-1703

June 17, 1971

MISTER

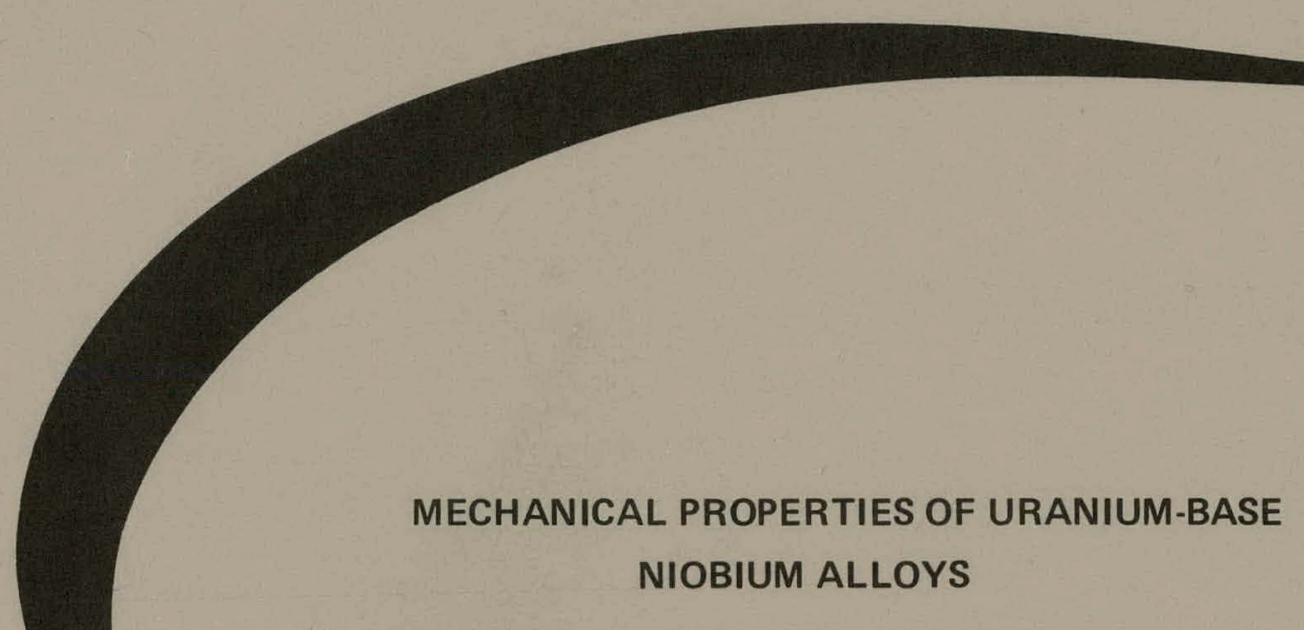

Ross J. Jackson and Joseph F. Boland

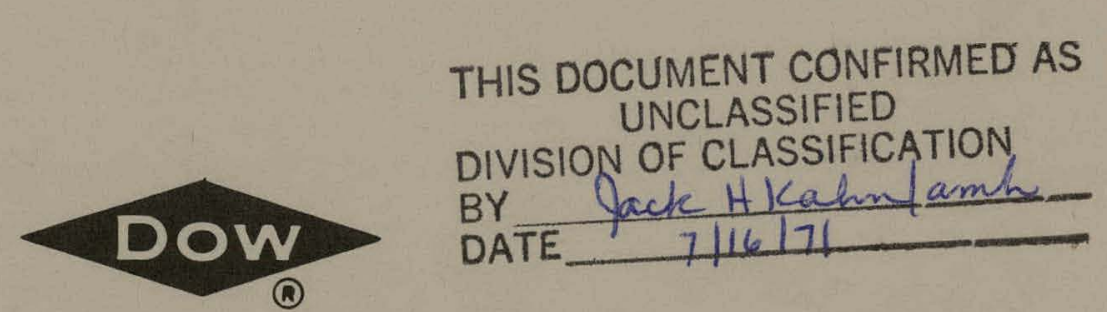

THE DOW CHEMICAL COMPANY

ROCKY FLATS DIVISION

P. O. BOX 888

GOLDEN, COLORADO 80401

U.S. ATOMIC ENERGY COMMISSION

CONTRACT AT(29-1)-1106 


\section{DISCLAIMER}

This report was prepared as an account of work sponsored by an agency of the United States Government. Neither the United States Government nor any agency Thereof, nor any of their employees, makes any warranty, express or implied, or assumes any legal liability or responsibility for the accuracy, completeness, or usefulness of any information, apparatus, product, or process disclosed, or represents that its use would not infringe privately owned rights. Reference herein to any specific commercial product, process, or service by trade name, trademark, manufacturer, or otherwise does not necessarily constitute or imply its endorsement, recommendation, or favoring by the United States Government or any agency thereof. The views and opinions of authors expressed herein do not necessarily state or reflect those of the United States Government or any agency thereof. 


\section{DISCLAIMER}

Portions of this document may be illegible in electronic image products. Images are produced from the best available original document. 


\section{LEGAL NOTICE}

This report was prepared as an account of work sponsored by the United States Government. Neither the United States nor the United States Atomic Energy Commission, nor any of their employees, nor any of their contractors, subcontractors, or their employees, makes any warranty, expressed or implied, or assumes any legal liability or responsibility for the accuracy, completeness or usefulness of any information, apparatus, product or process disclosed, or represents that its use would not infringe privately owned rights.

Printed in the United States of America Available from the

National Technical Information Service

U. S. Department of Commerce Springfield, Virginia 22151

Price: Printed Copy \$3.00: Microfiche $\$ 0.65$ 


\section{MECHANICAL PROPERTIES OF URANIUM-BASE NIOBIUM ALLOYS}

Ross J. Jackson and Joseph F. Boland

This report was prepared as an account of work sponsored hy the United States Government. Neither the United States nor the United States Atomic Energy Commission, nor any of their employees, nor any of their contractors, subcontractors, or their employees, makes any warranty, express or implied, or assumes any legal liability or responsibility for the acciliacy, completeness or usefulness of any information, apparatus, product or process disclosed, or represents that its use would not infringe privately owned rights.

\section{THE DOW CHEMICAL COMPANY ROCKY FLATS DIVISION \\ P. O. BOX 888 \\ GOLDEN, COLORADO 80401 \\ Prepared under Contract AT(29-1)-1106}

for the

Albuquerque Operations Office

U. S. Atomic Energy Commission 
RFP-1703 


\section{CONTENTS}

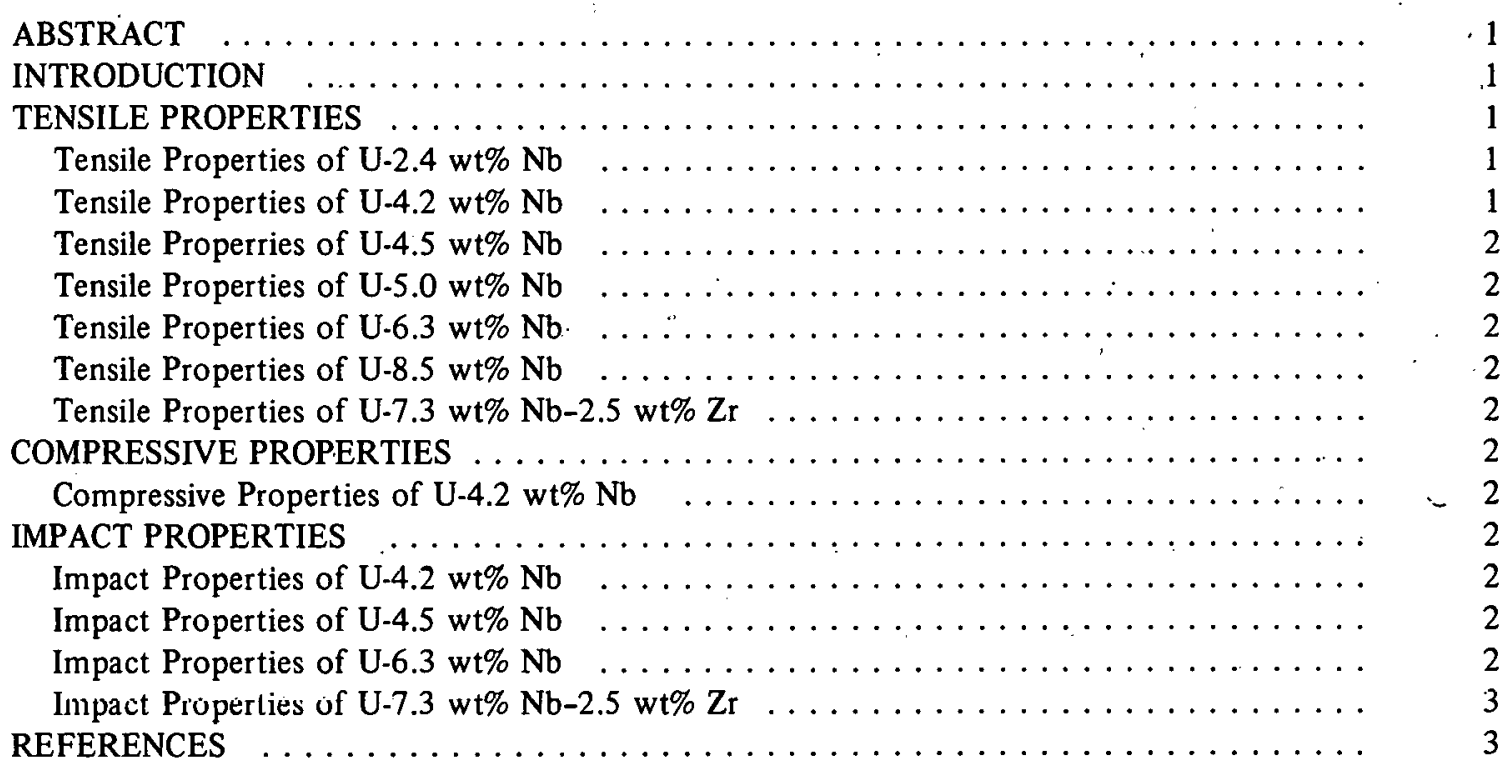


RFP-1703 


\title{
MECHANICAL PROPERTIES OF URANIUM-BASE NIOBIUM ALLOYS
}

\author{
Ross J. Jackson and Joseph F. Boland
}

\begin{abstract}
Tensile properties of gamma-quenched and aged binary U-2, U-4, U-5, U:6, and U-8 wt\% Nb alloys, and the ternary U-7.5 wt\% - $2.5 \mathrm{wt} \% \mathrm{Zr}$ alloy are presented. Two hundred sixty-four different combinations of compositiontime- temperature representing 527 tests are included. Impact properties of gamma-quenched and aged binary U-4 and U-6 wt\% Nb alloys, and the ternary U-7.5 Nb$2.5 \mathrm{Zr}$ alloy are presented. Thirty-six different combinations of composition-time-temperature representing 106 tests are included.
\end{abstract}

\section{INTRODUCTION}

This report summarizes mechanical property data generated at the Rocky Flats Plant on uranium-base niobium alloys and is current to April 1,1971. Two other Rocky Flats reports have dealt with mechanical properties of these alloys. Jackson and Miley ${ }^{1}$ discussed tensile properties of the quenched and aged U-4, U-6, and U-8 wt\% Nb alloys from a fundamental mechanistic point of view. From a similar point of view, Jackson and Boland ${ }^{2}$ discussed mechanical properties of the U-7.5 wt\% Nb$2.5 \mathrm{wt} \% \mathrm{Zr}$ alloy. For a discussion of the structures. and strengthening mechanism involved with uraniumbase niobium alloys the reader should consult the two above mentioned reports, since these subjects are not covered in this report.

Other reports dealing with mechanical properties of uranium-base niobium alloys are as follows. Zukas ${ }^{3}$ discussed the transformation kinetics and mechanical properties of the U-0.5 wt\% Nb-0.5 wt\% Mo alloy. Peterson and others ${ }^{4,5,6}$ studied physical and mechanical properties of numerous uranium alloy compositions, but prime interest was centered on the U.7.5 wt\% Nb-2.5 wt\% $\mathrm{Zr}$ alloy. Hoge ${ }^{7}$ investigated the effects of dynamic tension loading on the mechanical properties of the U.7.5 wt\% Nb-2.5 wt\% Ar alloy. Hoge found that tensile strength increased moderately with increasing strain rates $\left(10^{-3}\right.$ to 160 in./in.-sec.), but more important was the fact that dynamic loading caused only a slight decrease in ductility.

\section{TENSILE PROPERTIES}

Both cylindrical and flat tensile bars were used, and the dimensional specifications are listed in Figure 1. The type of bar used in a given test is specified in the figure caption. Spot checks for several fixed compositions and heat treatments using sister cylindrical and flat specimens or different size bars yielded near identical values for the measured tensile properties. Therefore, specimen geometry, within the limits of Figure 1, does not appear to alter measured tensile values.

Other test data such as composition, date of test, thermal and mechanical history, test temperature, strain rate, bars per datum point, etc., are also listed in the individual figure captions.

\section{Tensile Properties of U-2.4 wt\% Nb:}

Tensile test data for gamma-quenched U-2.4 wt\% $\mathrm{Nb}$ alloy aged 24 hours at various temperatures appear in Figure 2.

\section{Tensile Properties of U-4.2wt\% Nb:}

Figure 3 shows room temperature tensile test data for gamma-quenched and 80-hour aged U-4.2 wt\% $\mathrm{Nb}$ alloys. Figure 4 lists transverse and longitudinal room temperature tensile test data for gamma-quenched U.4.2 wt\% Nb alloys aged for 24 hours at various times. Figure 5 shows room temperature tensile test for gamma-quenched U-4.2 wt\% Nb alloys aged at $260^{\circ} \mathrm{C}$ for various times. Figure 6 lists comparative room temperature tensile data for gammaquenched U-4.2 wt\% $\mathrm{Nb}$ alloys aged at $260^{\circ} \mathrm{C}$ and $270^{\circ} \mathrm{C}$ for various times. There is no duplication of data in the four figures.

Tensile test bars longitudinal to the working direction (the alloy is worked prior to the $800^{\circ} \mathrm{C}$ heat treatment) show slightly different tensile properties than bars transverse (long transverse direction) to the working direction. This difference is shown in Figures 7 and 8 for two separate groups of hot forged gamma-quenched and $260^{\circ} \mathrm{C}$ and $270^{\circ} \mathrm{C} 80$-hour aged U-4.2 $\mathrm{Nb}$ alloys, respectively. The difference in longitudinal and transverse tensile values for two separate groups of U-4.2 wt\% Nb alloys aged 24 hours at $245^{\circ} \mathrm{C}$ is shown in Figure 9. The difference in the lwo groups is believed oaused by the anneal time at $800^{\circ} \mathrm{C}$ or to hydrogen content. 


\section{Tensile Properties of U-4.5 wt\% Nb:}

Figure 10 shows room temperature tensile-test data for gamma-quenched U-4.5 wt\% niobium alloy aged one hour at various times. Figures $11 \mathrm{a}$ and $11 \mathrm{~b}$ show comparative room temperature tensile test data for gamma-quenched U-4.5 wt $\% \mathrm{Nb}$ alloys aged at $250,260,270$, and $280^{\circ} \mathrm{C}$ for various times. The tensile data for the $250^{\circ}$ and $260^{\circ} \mathrm{C}$ age shown in Figures $11 \mathrm{a}$ and $11 \mathrm{~b}$ are plotted on a log time scale in Figure 12. Tensile test data for virgin arc-cast $\mathrm{U}-4.5 \mathrm{wt} \% \mathrm{Nb}$ aged 24 hours at various temperatures are shown in Figure 13.

\section{Tensile Properties of U -5.0 wt\% Nb:}

The 0.2\% offset yield strength as a function of composition (U-4.5 to U-5.1 wt\% $\mathrm{Nb}$ ) is shown in Figure 14.

\section{Tensile Properties of U-6.3 wt\% Nb:}

Figure 15 shows tensile test data for a U-6.3 wt\% Nb alloy aged one hour at various temperatures. Tensile test data for gamma-quenched U-6.3 wt\% $\mathrm{Nb}$ alloy aged at $150^{\circ} \mathrm{C}$ for various times appears in Figure 16. Hot tensile strength data for the U-6.3 wt\% $\mathrm{Nb}$ alloy aged two hours at a given temperature and then tested hot is shown in Figure 17.

\section{Tensile Properties of U-8.5 wt\% Nb:}

Tensile test data for gamma-quenched U-8.5 wt $\% \mathrm{Nb}$ aged one hour at various temperalures is shown in Figure 18.

\section{Tensile Properties of U-7.3 wt\% Nb-2.5 wt\% Zr:}

Figure 19 shows tensile test data for gamma-quenched U-7.3 Nb-2.5 $\mathrm{Zr}$ aged 80 hours at various temperatures. Figures 20a and 20b show similar data for the U.7.3 Nb$2.5 \mathrm{Zr}$ alloy aged 6 and 30 hours at various temperatures. Tensile test data for gamma-quenched U-7.3 Nb-2.5 Zr alloys aged at $200^{\circ} \mathrm{C}$ for various times is shown in Figure 21 .

\section{COMPRESSIVE PROPERTIES}

Compressive data on uranium-base niobium alloys are in short supply.
Compressive Properties of U-4.2 wt\% Nb:

Comparative compressive and tensile $0.2 \%$ offset yield strengths for the $800^{\circ} \mathrm{C}$ water quenched and aged $\mathrm{U}-4.2 \mathrm{wt} \% \mathrm{Nb}$ alloy are listed in Table 1. These alloys show a higher $0.2 \%$ offset yield strength in compression than in tension.

\begin{tabular}{|c|c|c|c|c|c|}
\hline Stren & ths. & & & & \\
\hline Composition: & U-4.2 wt \% & $6 \mathrm{Nb} \ldots$. . Bar & Type: 3 & $: 1, L: D$ & \\
\hline & Date: $4 / 7$ & $70 ; 11 / 70$ & & & \\
\hline Material: & Induction- & -cast recycle & , homog & enized $2 \mathrm{hr}$ & $\operatorname{ar} 1100^{\circ}$ \\
\hline & $\begin{array}{l}\text { hot forged } \\
\text { blanks ma }\end{array}$ & $\begin{array}{l}800^{\circ} \mathrm{C} \text {, wa } \\
\text { chined. }\end{array}$ & ter quen & ched, comp & ressive \\
\hline History: & & & $800^{\circ} \mathrm{C}$ & water quen & ched, \\
\hline & $\begin{array}{l}\text { aged } 80 \mathrm{hr} \\
\text { tested } 24^{\circ}\end{array}$ & $\begin{array}{l}\mathrm{r} \text { at } 260^{\circ} \mathrm{Co} \\
\mathrm{C} .\end{array}$ & $270^{\circ} \mathrm{C}$ & , air cooled & \\
\hline Strain Sagap: & SR-4. & & & & \\
\hline & & Compr & essive & Ten & ile \\
\hline Composition & $\begin{array}{c}\text { Heat } \\
\text { Treatment }\end{array}$ & $\begin{array}{c}0.2 \% \text { Y.S. } \\
\left(10^{3} \text { psi }\right)\end{array}$ & $\begin{array}{c}\text { No. of } \\
\text { Bars }\end{array}$ & $\begin{array}{c}0.2 \% \text { Y.S. } \\
\left(10^{3} \text { psi) }\right.\end{array}$ & $\begin{array}{c}\text { No. of } \\
\text { Bars }\end{array}$ \\
\hline $\mathrm{U}-4.2 \mathrm{wt} \% \mathrm{Nb}$ & $\begin{array}{l}\text { Aged } 80 \mathrm{hr} \\
\text { at } 260^{n} \mathrm{C}\end{array}$ & 176 & 4 & 163 & 3 \\
\hline $\mathrm{U}-4.2 \mathrm{wt} \% \mathrm{Nb}$ & $\begin{array}{l}\text { Aged } 80 \mathrm{hr} \\
\text { at } 270^{\circ} \mathrm{C}\end{array}$ & 189 & 4 & 174 & 32 \\
\hline
\end{tabular}

\section{IMPACT PROPERTIES}

Standard V-notch Charpy impact specimens were used. Test data such as composition, date of test, thermal and mechanical history, test temperature, bar's per datum point, ctc., arc listed in the Indtvidual flgure urulinins.

\section{Impact Properties of U-4.2 wt\% Nb:}

Charpy V-notch impact strengths for gamma-quenched $\mathrm{U}-4.2 \mathrm{wt} \% \mathrm{Nb}$ aged 80 hours at $270^{\circ} \mathrm{C}$ and then tested at temperature are shown in Figure 22.

\section{Impact Properties of U-4.5 wt\% Nb:}

Room temperature, Charpy $V$-nutch inpact strengths for gamma-quenched U-4.5 $\mathrm{wt} \% \mathrm{Nb}$ alloys aged one hour at various temperatures appear in Figure 23.

\section{Impact Properties of U-6.3 wt\% Nb:}

Room temperature, Charpy V-notch impact strengths for gamma-quenched (both water and air quenched) are shown in Figure 24. Impact strengths as a function of density (niobium content) appear in Figure 25. 


\section{Impact Properties of U-7.3 wt\% Nb-2.5 wt\% Zr:}

Impact test data (Charpy V-notch) for gamma-quenched U-7.3 wt\% Nb-2.5 wt\% $\mathrm{Zr}$ aged 30 hours at various temperatures appear in Figure 26. Impact test data (Charpy V-notch) for gamma-quenched U-7.3 Nb$2.5 \mathrm{Zr}$ aged at $200^{\circ} \mathrm{C}$ for various times is shown in Figure 27.

\section{REFERENCES}

1. R. J. Jackson and D. V. Miley, "Tensile Properties of Gamma Quenched and Aged Uranium-Base Niobium Alloys," ASM Trans. Quart., 61, 336-343, 1968.

2. R. J. Jackson and J. F. Boland, "Mechanical Properties of the Uranium-7.5 Niobium-2.5 Zirconium Ternary Alloy," USAEC Report RFP-1652, Rocky Flats Division, The Dow Chemical Company, 1971.
3. E. G. Zukas, "Transformation Kinetics and Mechanical Properties of Uranium- 0.5 wt\% Nb-0.5 wt\% Mo Alloy," Trans $A S M$, 52: 797, 1960.

4. C. A. W. Peterson and R. R. Vandervoort, "Mechanical Properties of Some Uranium Alloys," USAEC Report UCRL-7771 (Lawrence Radiation Laboratory, Livermore, California), 1964.

5. C: A. W. Peterson and R. R. Vandervoort, "Properties of a Metastable Gamma-Phase Uranium-Base Alloy: U-7.5 Nb2.5 Zr," USAEC Report UCRL-7869 (Lawrence Radiation Laboratory, Livermore, California), 1964.

6. C. A. W. Peterson and W. E. Elkington, "Physical and Mechanical Properties of U-7.5 wt\% Nb-2.5 wt\% Zr Alloy," USAEC Report UCRL-14724 (Lawrence Radiation Laboratory, Livermore, California), 1966.

7. K. G. Hoge, "Mechanical Properties of Gamma Phase Uranium-7.5 wt\% Niobium-2.5 wi \% Zirconium Alloy Under Dynamic Loading," USAEC Report UCRL-14805 (Lawrence Radiation Laboratory, Livermore, California), 1966. 
RFP-1703
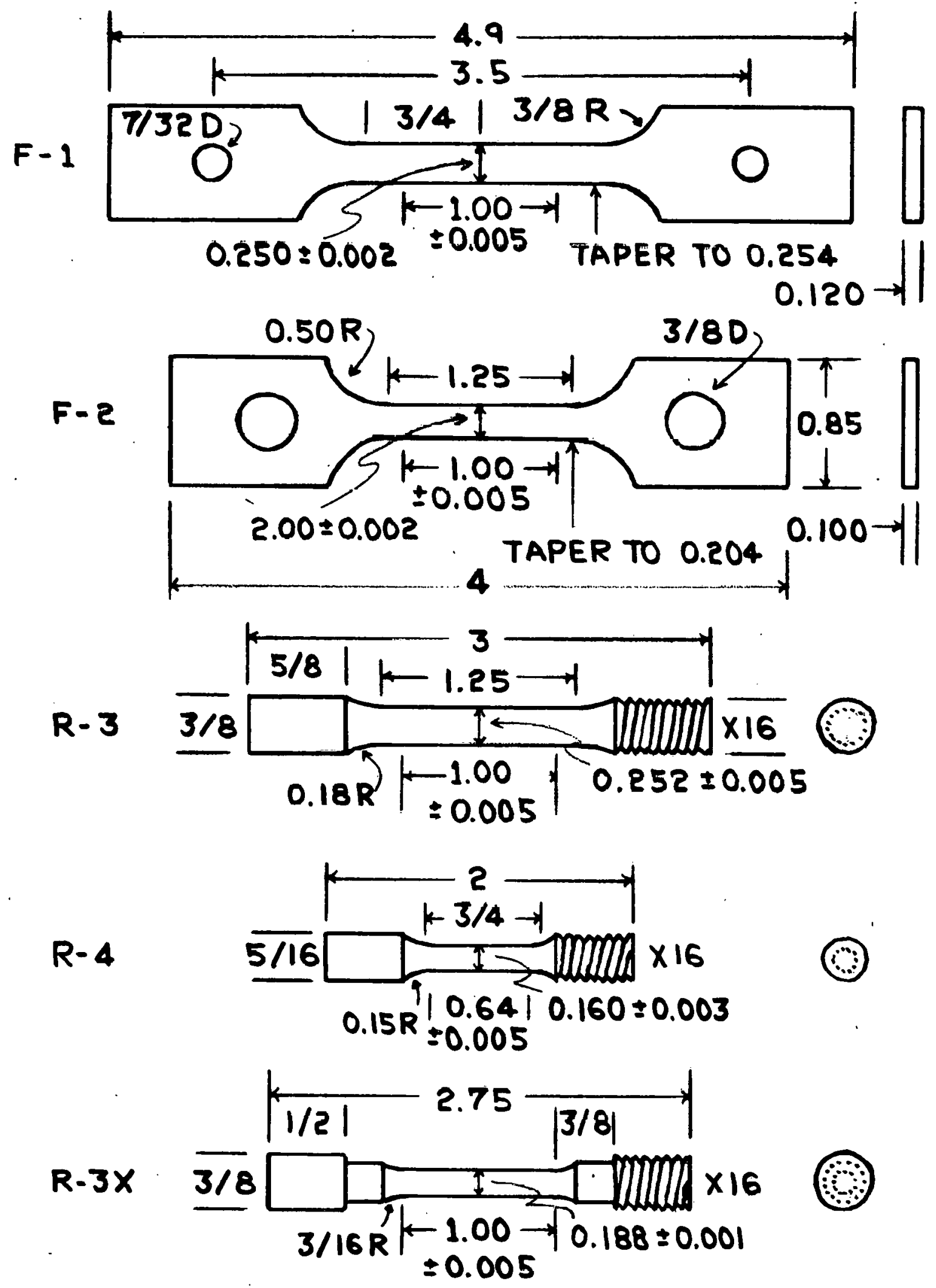

(a)

Figure 1. Design and Specification of Tensile Bars Used In This Study. 

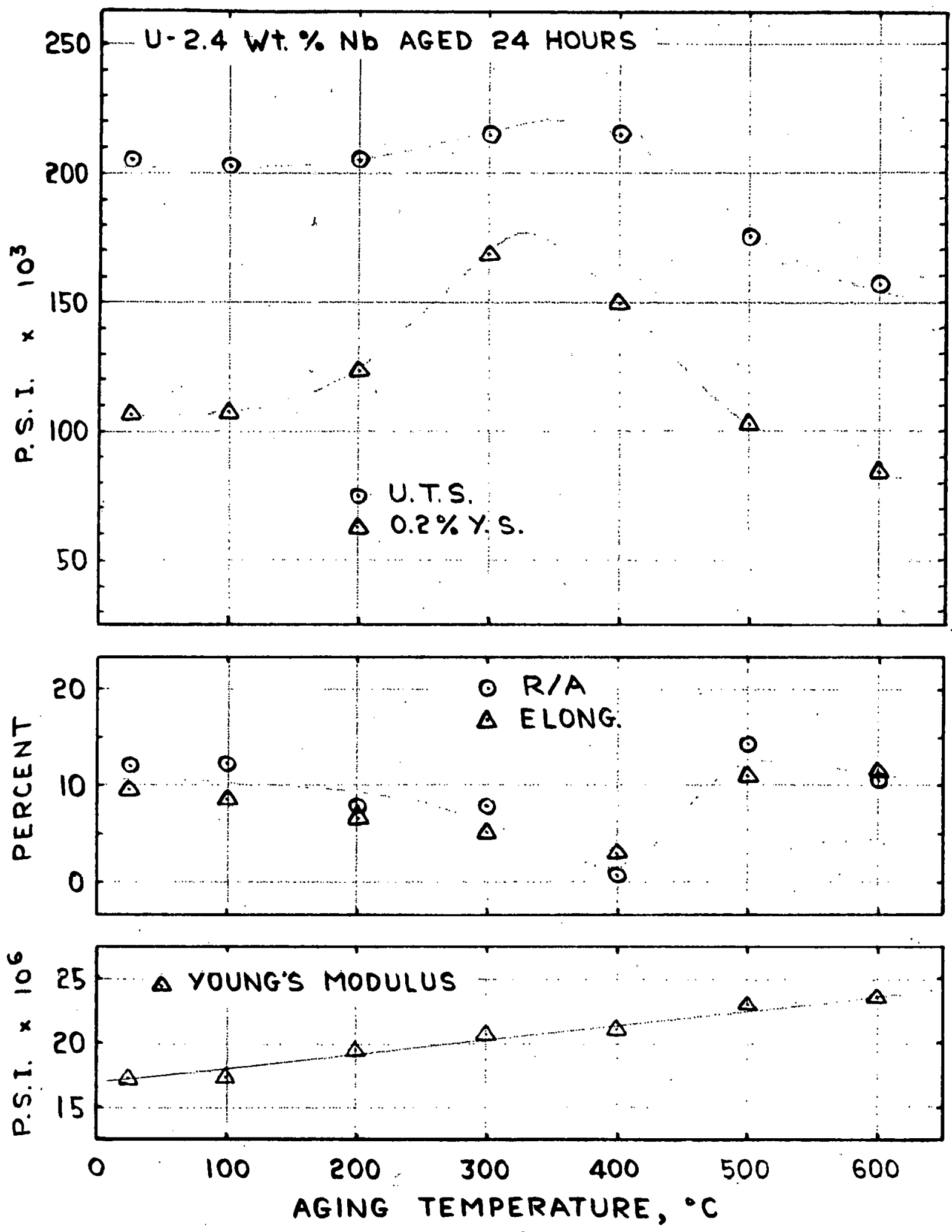

Figure 2. Tensile Test Data for 24 Hour Aged U-2.4 Nb Alloy.

Composition: U-2.4 wt\% Nb. ... Bar Type: R-4. . . Date: $2 / 71$.

Material: Single-arc-cast virgin, hot forged $820^{\circ} \mathrm{C}$, homogenized $2 \mathrm{hr}$ at $1050^{\circ} \mathrm{C}$, hot rolled $620^{\circ} \mathrm{C}$, rough tensile blanks machined.

History: Gammaized $20 \mathrm{~min}$. at $820^{\circ} \mathrm{C}$, water quenched, aged $24 \mathrm{hr}$ at temperature, air cooled, machined, tested $24^{\circ} \mathrm{C}$.

Strain Rate: $\quad 0.005$ in./in.-min. ... Bars' per datum point: 2. 

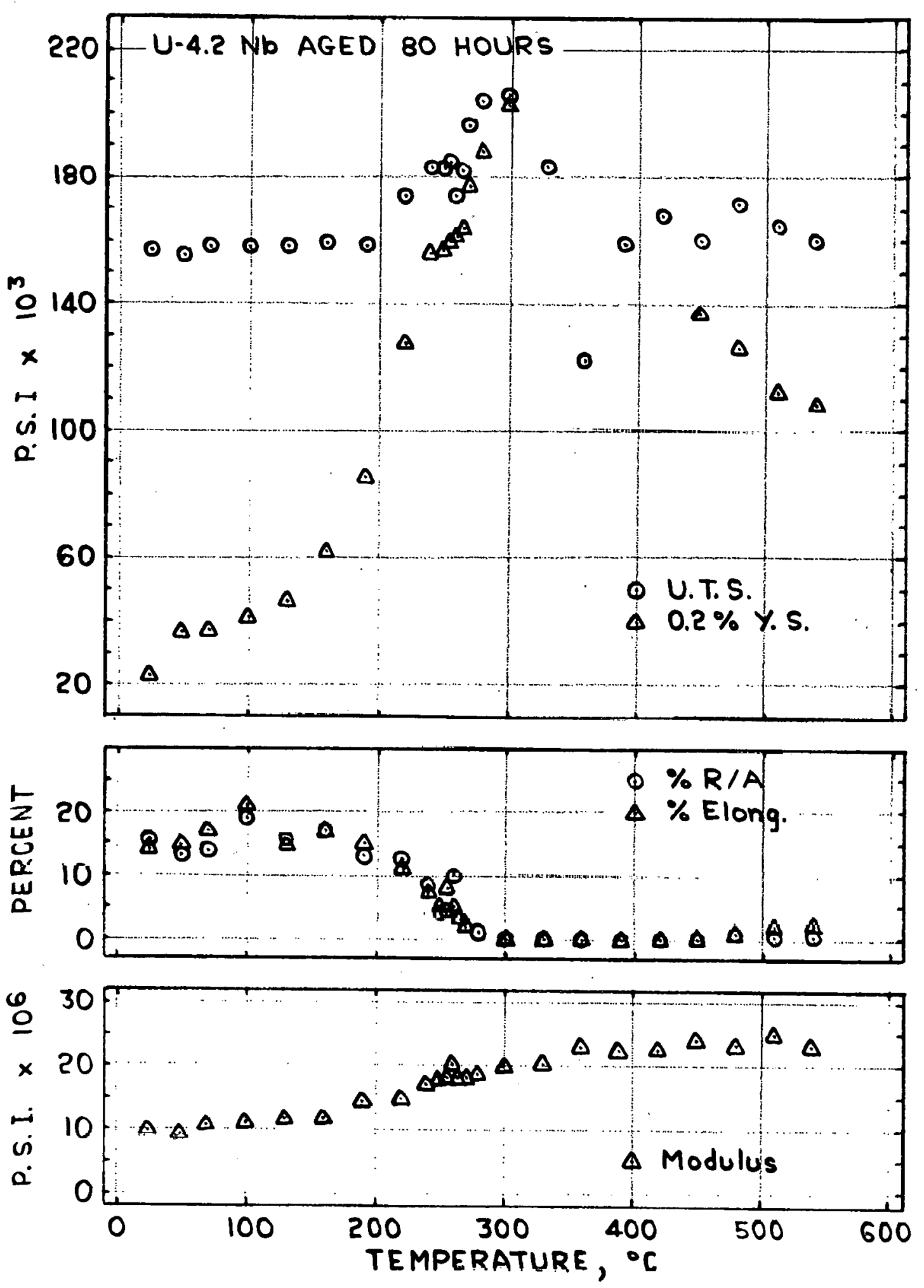

Figure 3. Tensile Test Data for 80 Hour Aged U-4.2 Nb Alloy.

Composition: U-4.2 wt\% Nb. . . Bar Type: R-3... Date: $3 / 70$.

Material: Induction-cast recycle, homogenized $4 \mathrm{hr}$ at $1100^{\circ} \mathrm{C}$, hot forged $800^{\circ} \mathrm{C}$, water quenched, tensile blanks machined.

History: Gammaized $30 \mathrm{~min} .800^{\circ} \mathrm{C}$, water quenched, aged $80 \mathrm{ht}$ at temperature, air cooled, machincd, tested $24^{\circ} \mathrm{C}$.

Strain Rate: 0.005 in./in.-min. . . Bars per datum point: 1. 

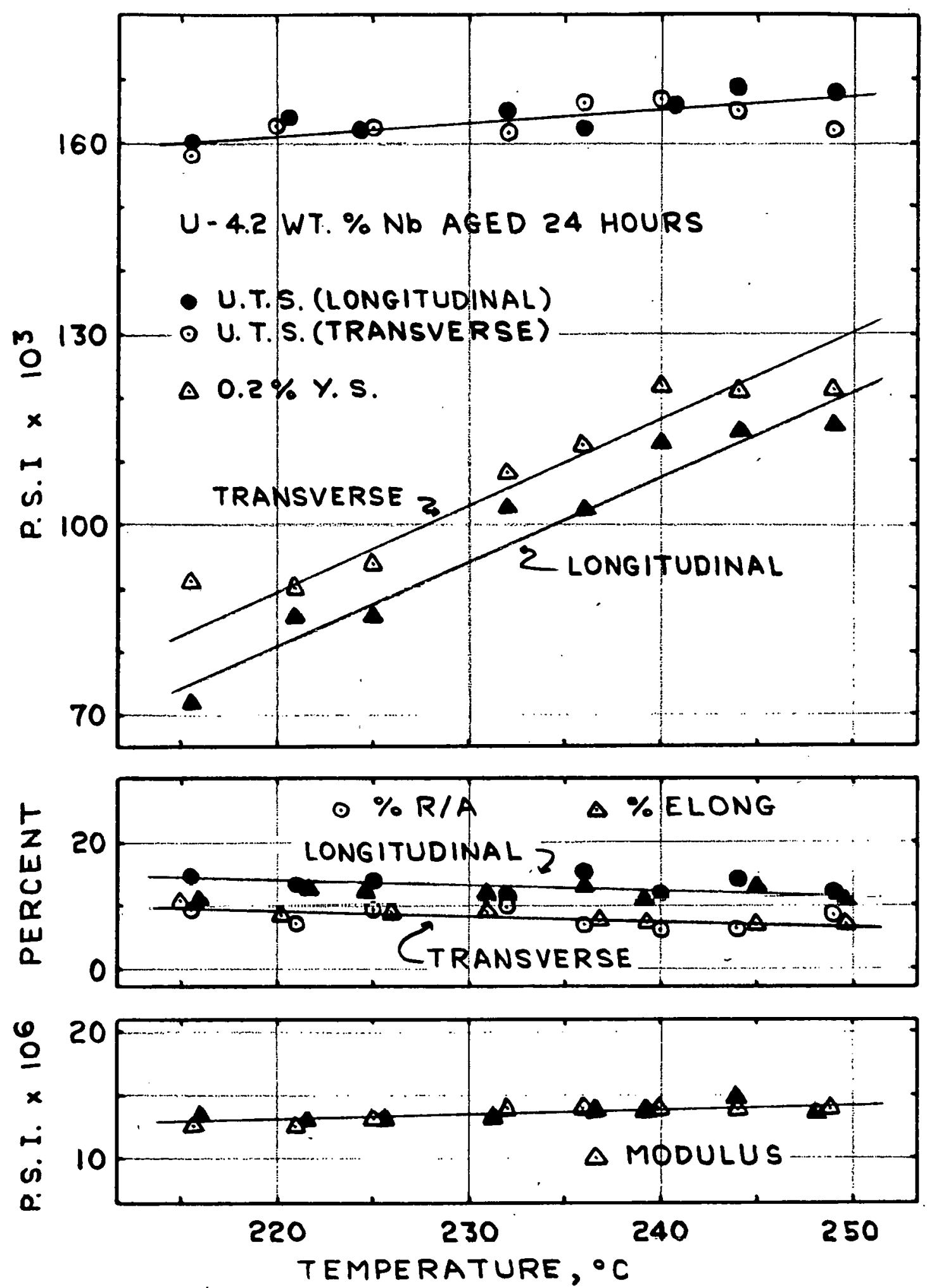

Figure 4. Longitudinal and Transverse Tensile Data for U-4.2 Nb Aged 24 Hours.

Composition: U-4.2 wt\% Nb. ... Bar Type: R-4... Date: $1 / 71$.

Material: Induction-cast recycle, homogenized $4 \mathrm{hr}$ at $1100^{\circ} \mathrm{C}$, hot extruded $800^{\circ} \mathrm{C}$, water quenched, tensile blanks machined transverse and longitudinal to extrusion direstion.

Hiștory: Gammaized $30 \mathrm{~min} .800^{\circ} \mathrm{C}$, water quentilied, aged $24 \mathrm{hr}$ at vartous tempèratures, air cooled, machined, tested $24^{\circ} \mathrm{C}$. Strain Rate: 0.005 in./in.-min. . . Bars per datum point: 2. 


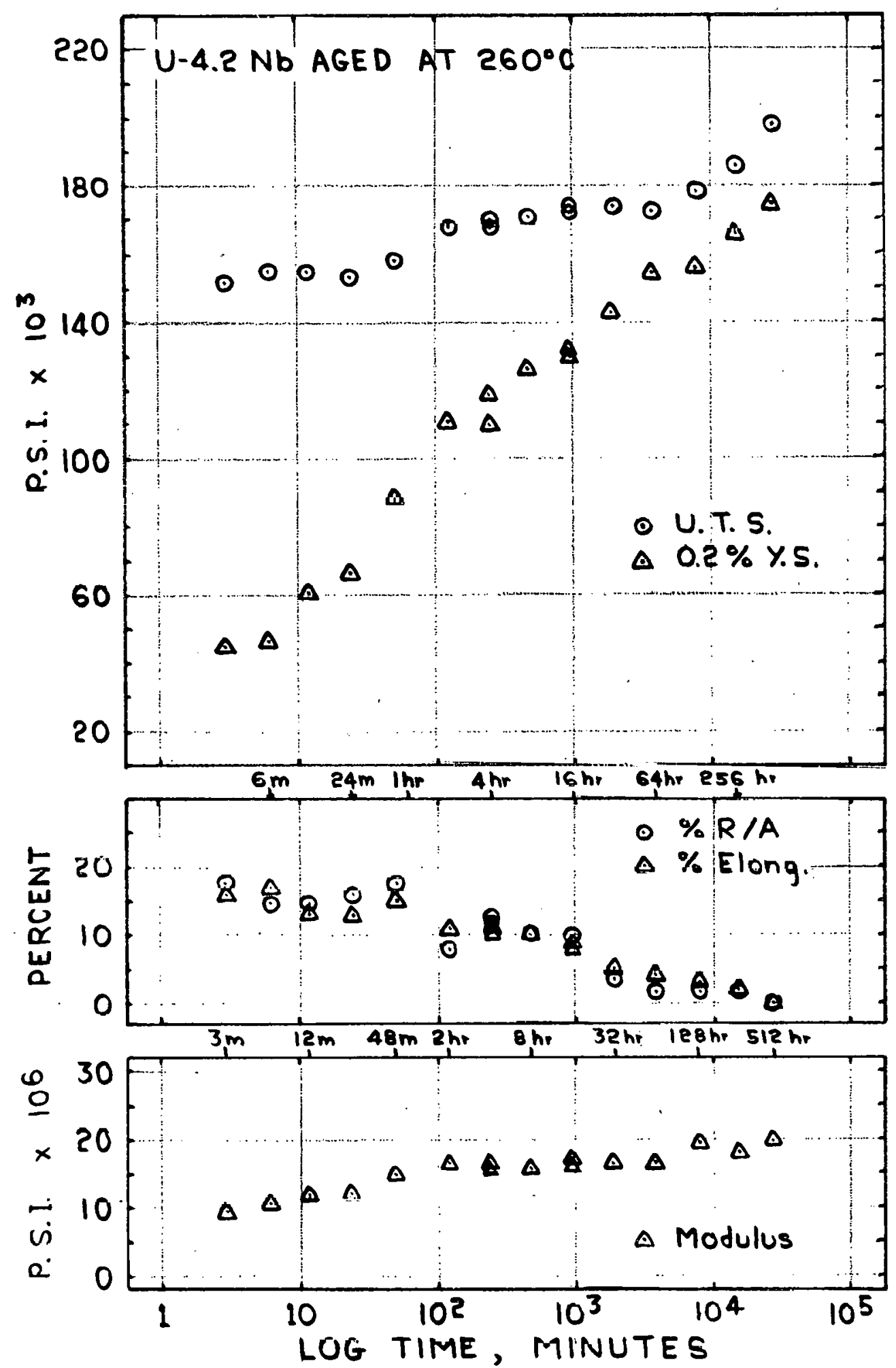

Figure 5. Tensile Test Data for $260^{\circ} \mathrm{C}$ Aged U-4.2 Nb Alloy.

Material: Induction-cast recycle, homogenized $4 \mathrm{hr}$ at $1100^{\circ} \mathrm{C}$, hot extruded $800^{\circ} \mathrm{C}$, water quenched, tensile blanks machined.

History: Gammaized $30 \mathrm{~min} .800^{\circ} \mathrm{C}$, water quenched, aged $260^{\circ} \mathrm{C}$ for various times, air cooled, machined, tested $24^{\circ} \mathrm{C}$.

Strain Rate: 0.005 in./in.-min. . . Bars per datum point: 1 . 


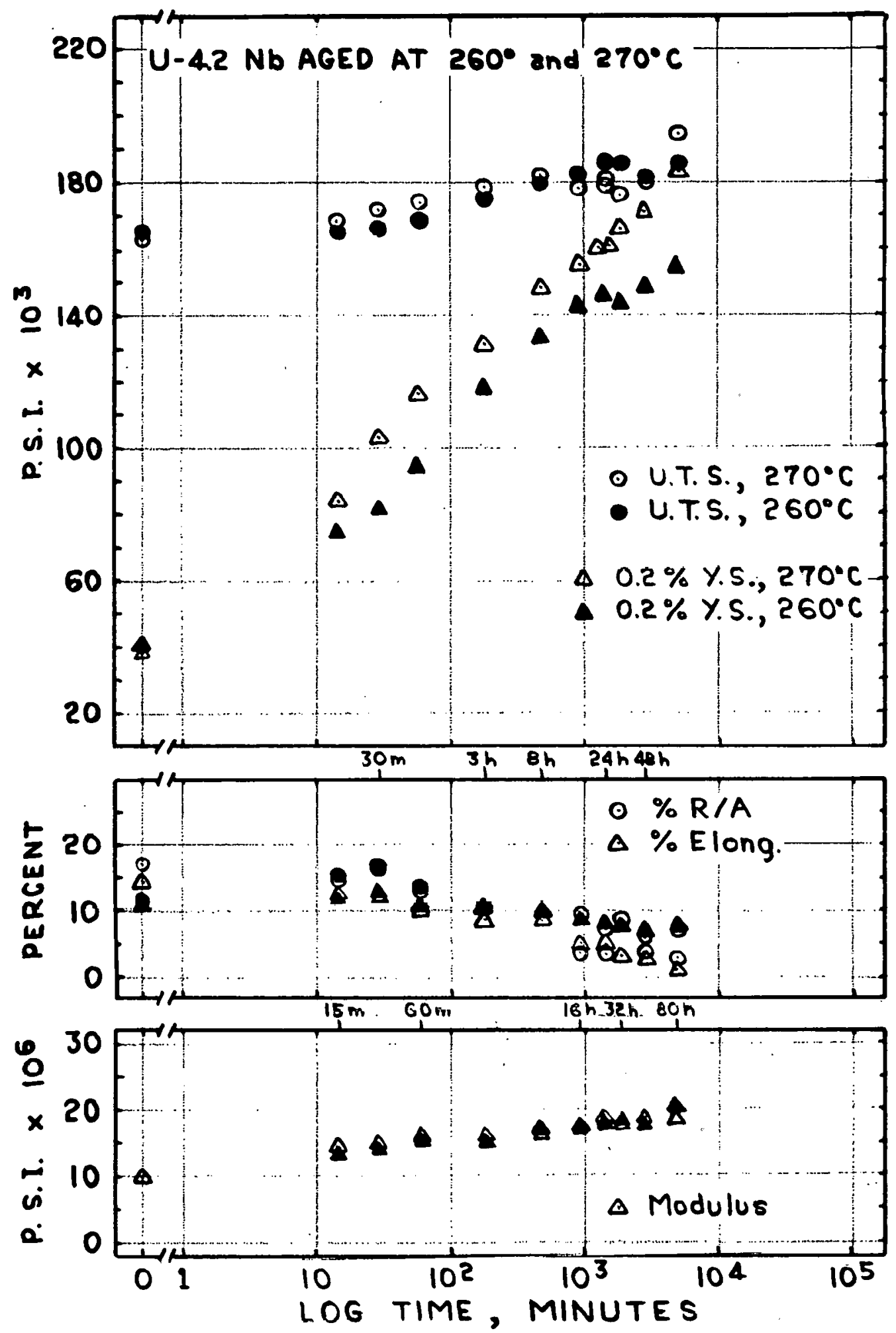

Figure 6. Tensile Test Data for $260^{\circ}$ and $270^{\circ} \mathrm{C}$ Aged U-4.2 Nb Alloy.

Composition: U-4.2 wt\% Nb. ... Bar Type: R-3... Date: $9 / 68$.

Material: Induction-cast recycle, homogenized $2 \mathrm{hr}$ at $1100^{\circ} \mathrm{C}$, hot extruded $800^{\circ} \mathrm{C}$, water quenches, tensile hlanks machined.

History: Gammaized $30 \mathrm{~min} .800^{\circ} \mathrm{C}$, water quenched, aged $260^{\circ} \mathrm{C}$ or $270^{\circ} \mathrm{C}$ for various times, air cooled, machined, tested $24^{\circ} \mathrm{C}$.

Strain Rate: 0.005 in./in.-min. ... Bars per datum point: 1 . 


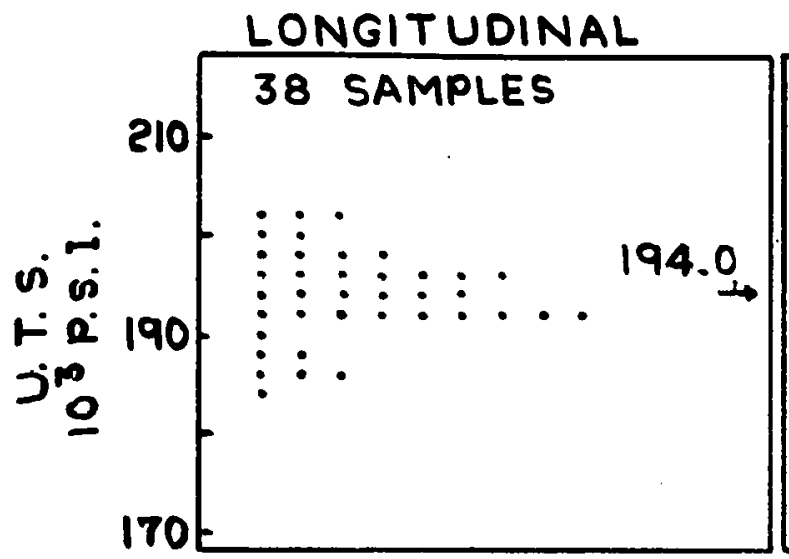

TRANSVERSE
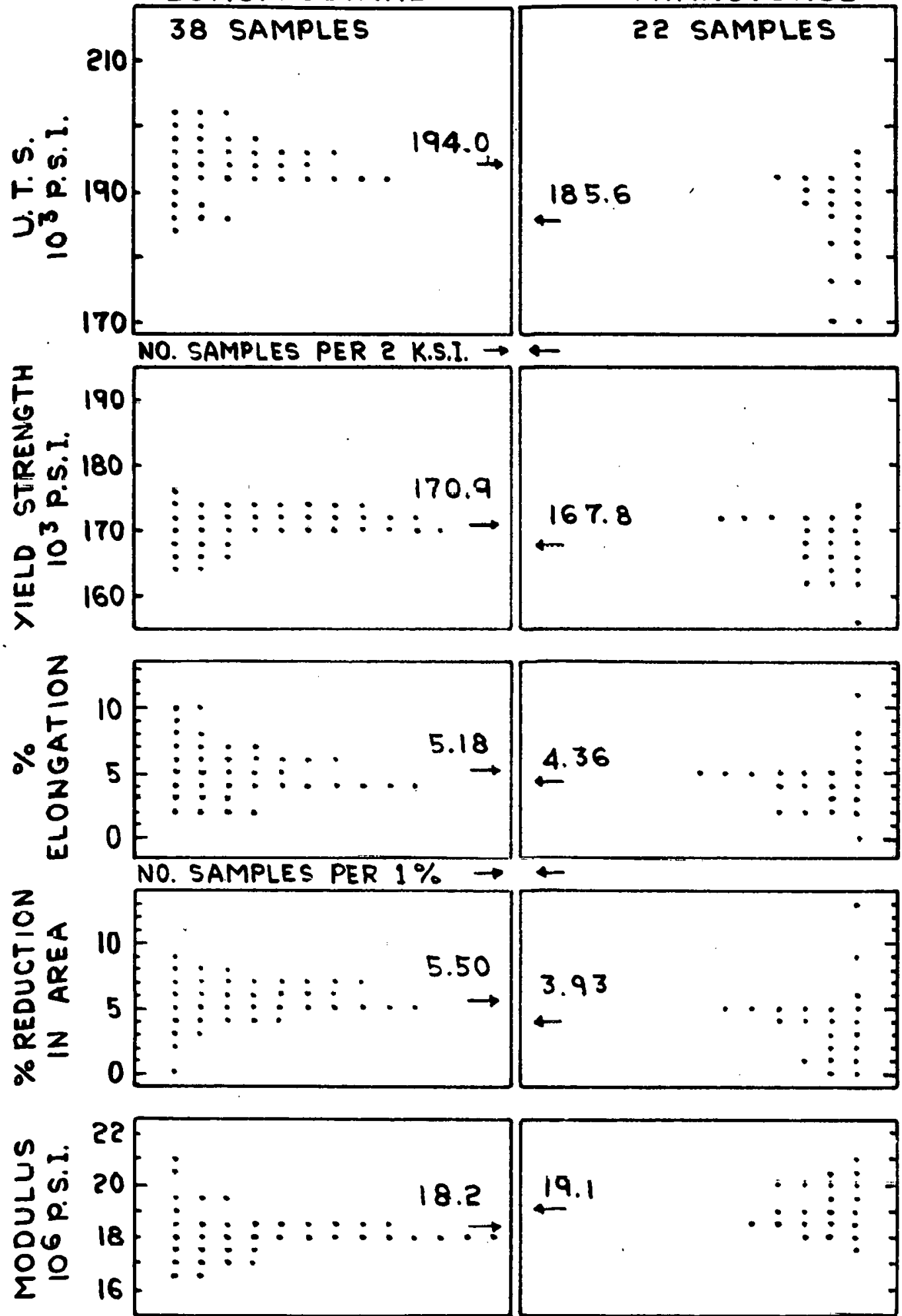

Figure 7. Longitudinal and Transverse Tensile Data for U-4.2 Nb Aged 80 Hours at $260^{\circ} \mathrm{C}$.

Composition: U-4.2 wt\% Nb. . . Bar Type: R-4... Date: $7 / 69$.

Material: Induction-cast recycle, homogenized $4 \mathrm{hr}$ at $1100^{\circ} \mathrm{C}$, hot-forged $800^{\circ} \mathrm{C}$, water quenched, tensile blanks machined transversc and longitudinal to working direction.

History: Gammaized $20-60 \mathrm{~min} .800^{\circ} \mathrm{C}$, water quenched, aged $80 \mathrm{hr}$ at $260^{\circ} \mathrm{C}$, air cooled, machined, tested $24^{\circ} \mathrm{C}$. Strain Rate: $\quad 0.005$ in./in.-min. ... Bars per datum point: 1 . 

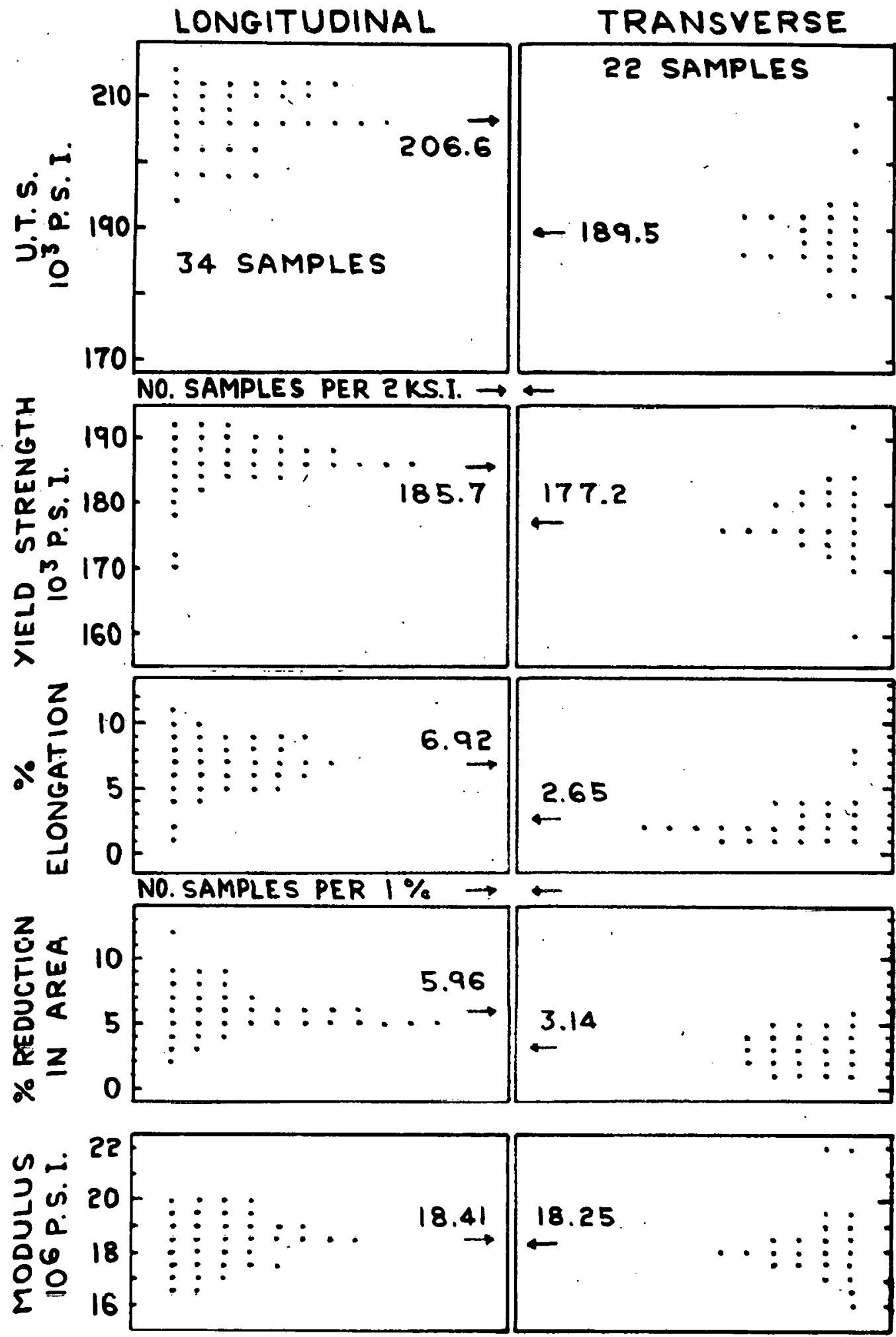

Figure 8. Longitudinal and Transverse Tensile Data for U-4.2 Nb Aged 80 Hours at $260^{\circ} \mathrm{C}$.

Composition: U-4.2 wt\% Nb. . . Bar Type: R-4. . . Date: $7 / 69$.

Material: Induction-cast recycle, homogenized $4 \mathrm{hr}$ at $1100^{\circ} \mathrm{C}$, hot-forged $800^{\circ} \mathrm{C}$, water quenched, tensile blanks machined transverse and longitudinal to working direction.

History: Gammaized $20.60 \mathrm{~min} .800^{\circ} \mathrm{C}$, watel quericlied, aged $80 \mathrm{hr}$ at $270^{\circ} \mathrm{C}$, air coóled, machined, tested $24^{\circ} \mathrm{C}$.

Strain Rate: 0.005 in./in.-min. ... Bars per datum point: 1 . 
RFP-1703
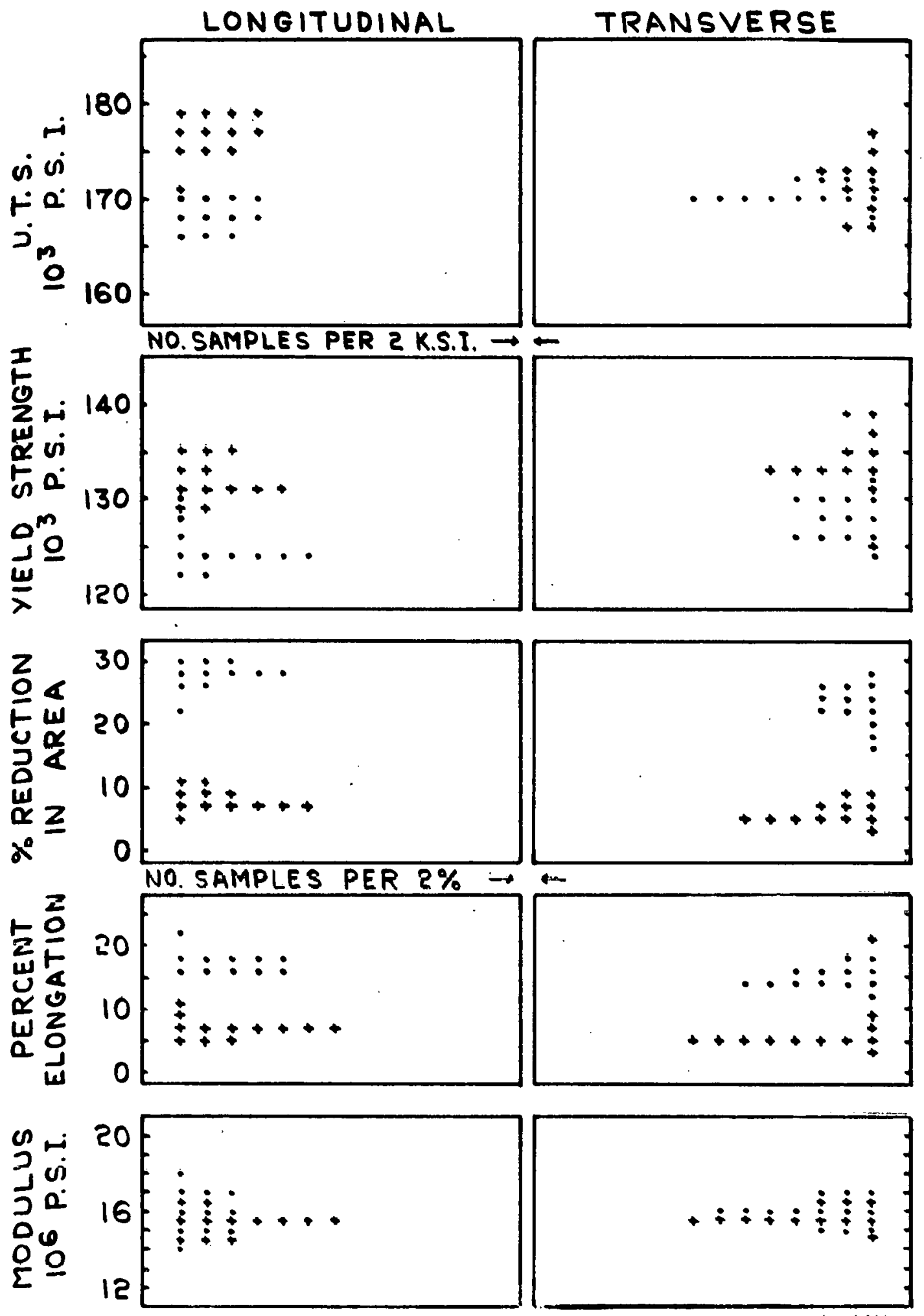

Figure 9. Longitudinal and Transverse Tensile Data for U-4.2 Nb Aged 24 Hours at $245^{\circ} \mathrm{C}$.

Composition: U-4.2 wt\% Nb. . . Bar Type: R-4... Date: $1 / 71,3 / 71$.

Material: Induction-cast recycle, homogenized $2 \mathrm{hr}$ at $1100^{\circ} \mathrm{C}$, hot extruded $800^{\circ} \mathrm{C}$, water quenched, tensile blanks machined transverse and longitudinal to extrusion direction.

History: Gammaized 15 or $45 \mathrm{~min} .800^{\circ} \mathrm{C}$, water quenched, aged $24 \mathrm{hr}$ at various temperatures, air cooled, machined, tested $24^{\circ} \mathrm{C}$. Strain Rate: $\quad 0.005$ in./in.-min. ... Bars per datum point: 1 . 
U. $4.5 \mathrm{Wt} \% \mathrm{Nb}$ AGED 1 HOUR
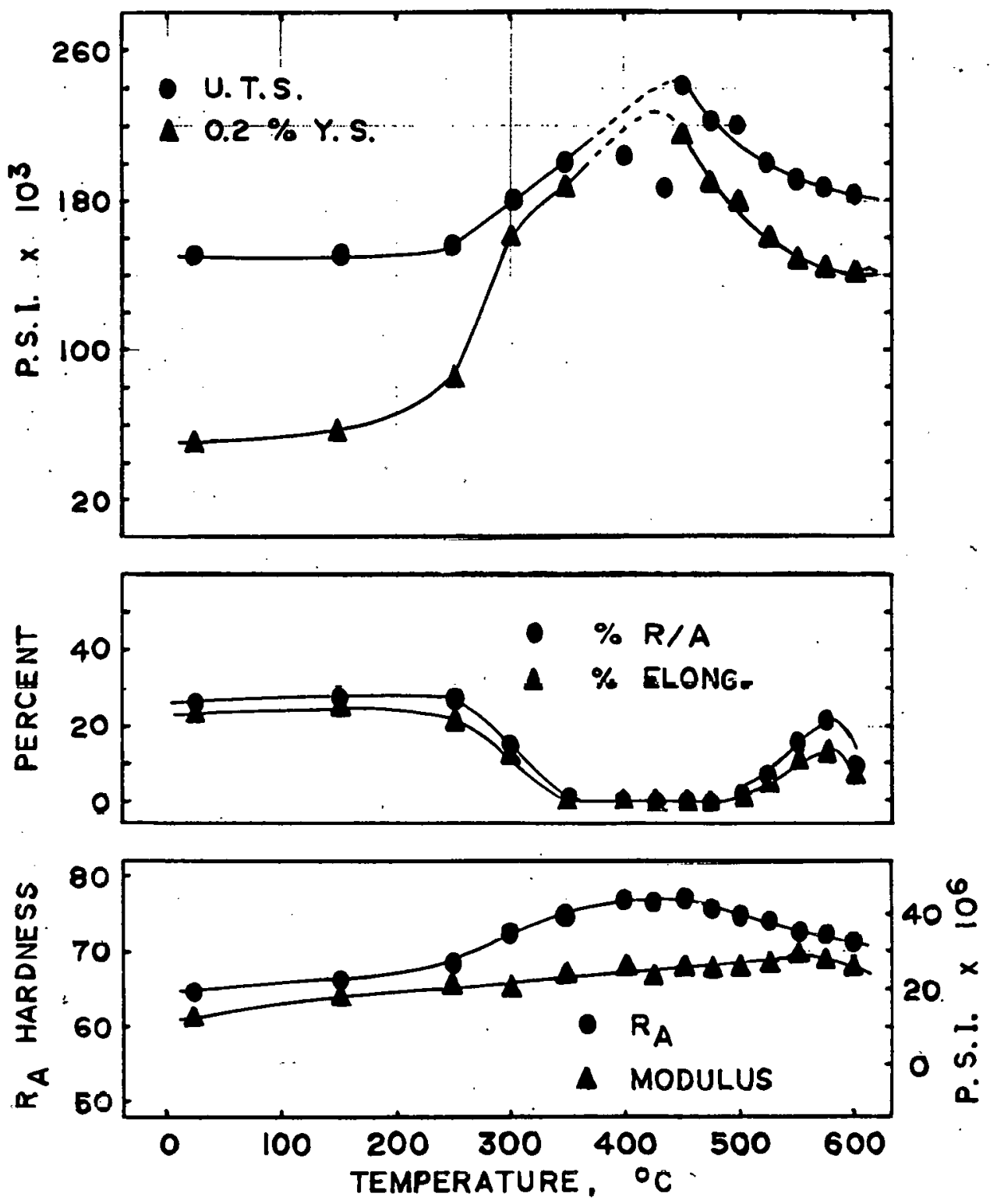

Figure 10. Tensile Test Data for 1 Hour Aged U-4.5 Nb Alloy.

Composition: U-4.5 wt\% Nb. . . Bar Type: F-1... Date: $10 / 66$.

Material: Double arcesst virgin, homogenized $2 \mathrm{hr}$ at $1100^{\circ} \mathrm{C}$, hot rolled $800^{\circ} \mathrm{C}$, wale, yuenched, tenslle blanks machined.

Histury: Gammaized 3ủ min. at $8 \mathrm{8U}^{\circ} \mathrm{C}$, water quenched, aged $1 \mathrm{hr}$ at various temperatures, air cooled, machined, tested $24^{\circ} \mathrm{C}$. Strain Rate: $\quad 0.002$ in./in.-min. . . Bars per datum point: 3. 

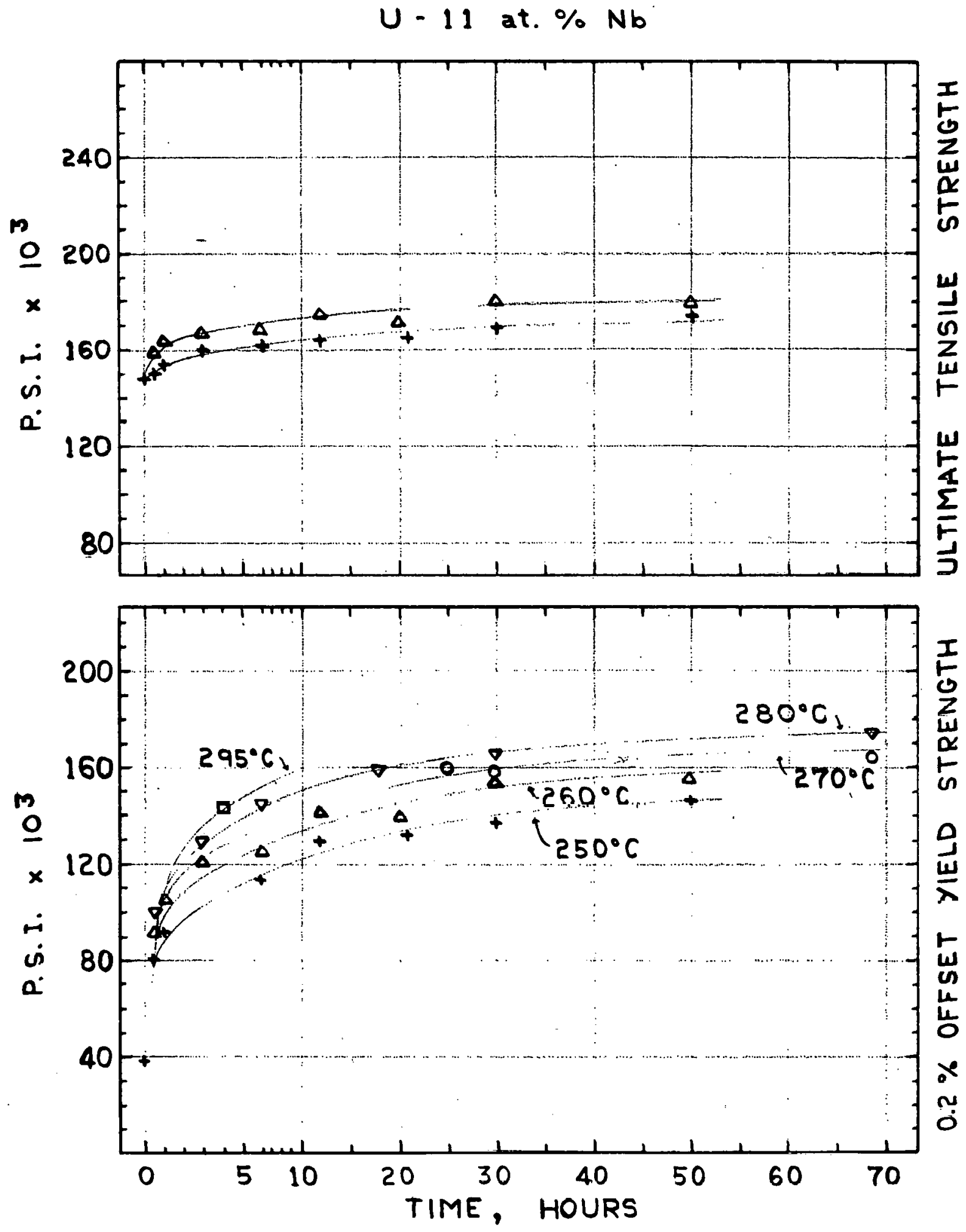

Figure 11a. Tensile Test Data for $250,260,270,280$, and $295^{\circ} \mathrm{C}$ Aged U-4.5 Nb Alloy.

Composition: U-4.5 wt\% Nb. . . Bar Type: R-3. . . Date: $4 / 68$.

Material: Induction-cast recycle, homogenized $2 \mathrm{hr}$ at $1100^{\circ} \mathrm{C}$, hot forged $800^{\circ} \mathrm{C}$, water quenched, tensile blanks machined.

History: Gammaized $30 \mathrm{~min} .800^{\circ} \mathrm{C}$, water quenched, aged $250,260,270,280$, and $295^{\circ} \mathrm{C}$ for various times, air cooled, machined, tested $24^{\circ} \mathrm{C}$.

Strain Rate: $\quad 0.005$ in./in.-min. ... Bars per datum point: 1 . 


\section{$U-\mid 1$ at. $\% \mathrm{Nb}$}
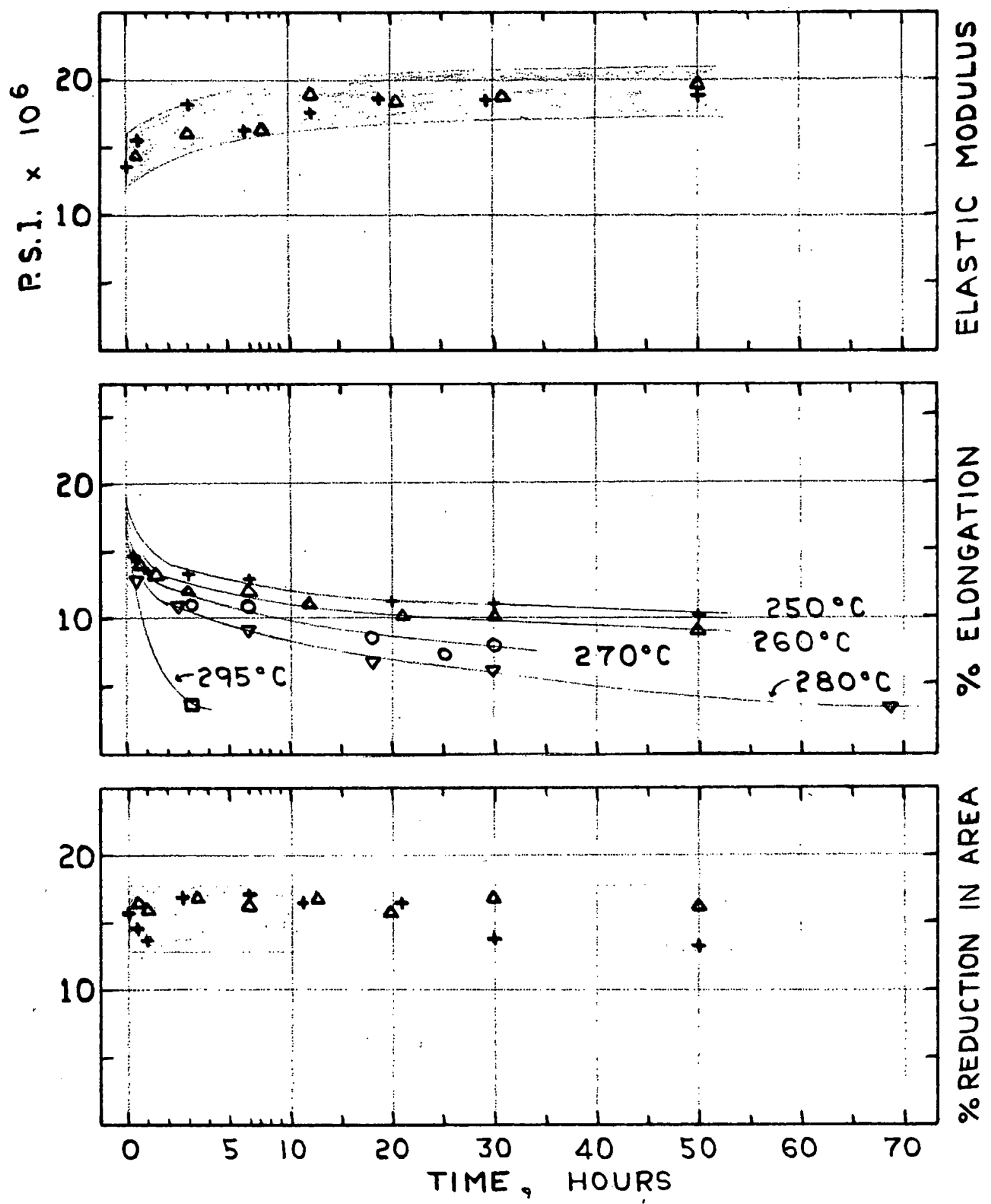

Figure 11b. Tensile Test Data for $250,260,270,280$, and $295^{\circ} \mathrm{C}$ Aged U-4.5 $\mathrm{Nb}$ Alloy.

Composition: U-4.5 wt\% Nb. . . Bar Type: R-3... Date: $4 / 68$.

Material: Induction-cast recycle, homogenized $2 \mathrm{hr}$ at $1100^{\circ} \mathrm{C}$, hot forged $800^{\circ} \mathrm{C}$, water quenched, tensile blanks machined.

History: Gammaized $30 \mathrm{~min} .800^{\circ} \mathrm{C}$, water quenched, aged $250,260,270,280$, and $295^{\circ} \mathrm{C}$ for various times, air cooled, machined, tested $24^{\circ} \mathrm{r}$

Etrain Rate: $\quad 0.005$ in./ln.-min. . . Bars per datuini point: 1 . 
RFP-1703
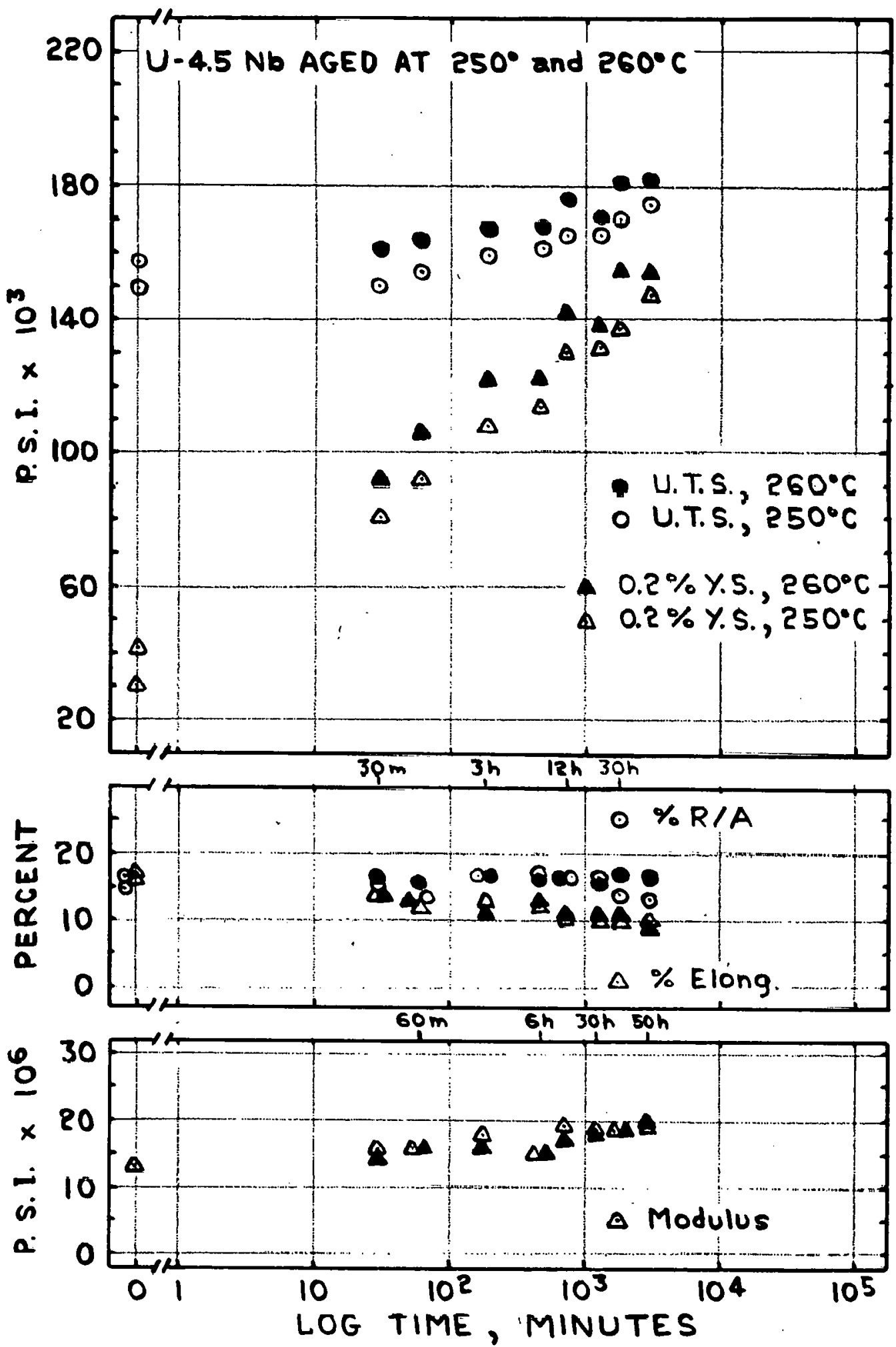

Figure 12. Tensile Data for $250^{\circ} \mathrm{C}$ and $260^{\circ} \mathrm{C}$ Aged U-4.5 Nb Alloy.

Composition: U-4.5 wt\% Nb. ... Bar Type: R-3: .. Date: $4 / 68$.

Material: Induction-cast recycle, homogenized $2 \mathrm{hr}$ at $1100^{\circ} \mathrm{C}$, hot forged $800^{\circ} \mathrm{C}$, water quenched, tensile blanks machined.

History: Gammaized $30 \mathrm{~min} .800^{\circ} \mathrm{C}$, water quenched, aged 250 and $260^{\circ} \mathrm{C}$ for various times, air cooled, machined, tested $24^{\circ} \mathrm{C}$. Strain Rate: $\quad 0.005$ in./in.-min. . . Bars per datum point: 1 . 

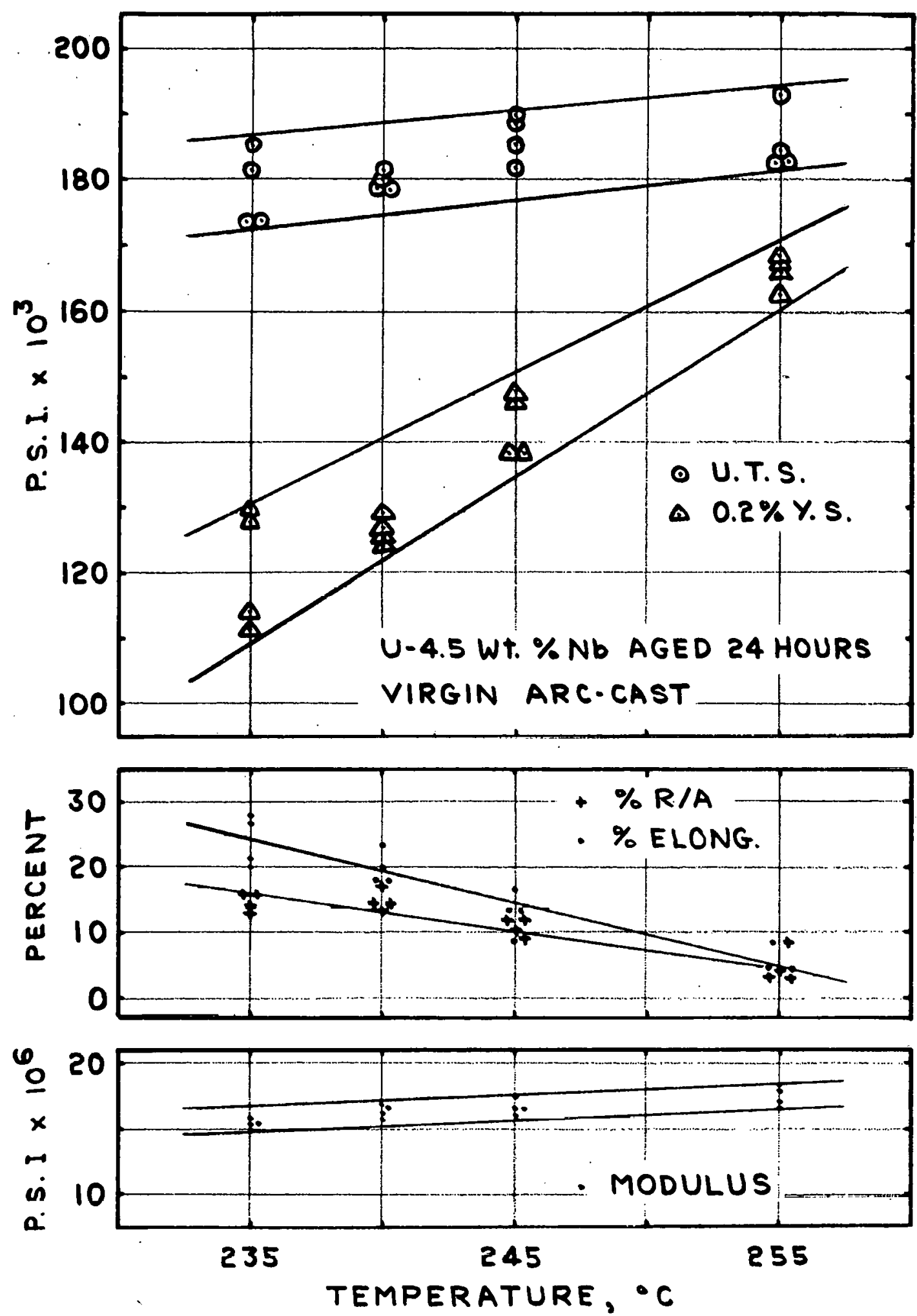

Figure 13. Tensile Test Data for Virgin, Arc-cast U-4.5 Nb Aged 24 Hours.

Composition: U-4.5 wt\% Nb. . . Bar Type: R-4... Date: $1 / 71$.

Material: Double arc-cast virgin, homogenized $4 \mathrm{hr}$ at $1100^{\circ} \mathrm{C}$, hot forged $800^{\circ} \mathrm{C}$, water quenched, tensile blanks machined.

History: Gammaized $30 \mathrm{~min}$. at $800^{\circ} \mathrm{C}$, water quonohod, agod $31 \mathrm{hr}$ at various tomporaturos, air coolod, machined, tested $24^{\circ} \mathrm{C}$. Strain Rate: 0.005 in./in.-min. ... Bars per datum point: 1. 


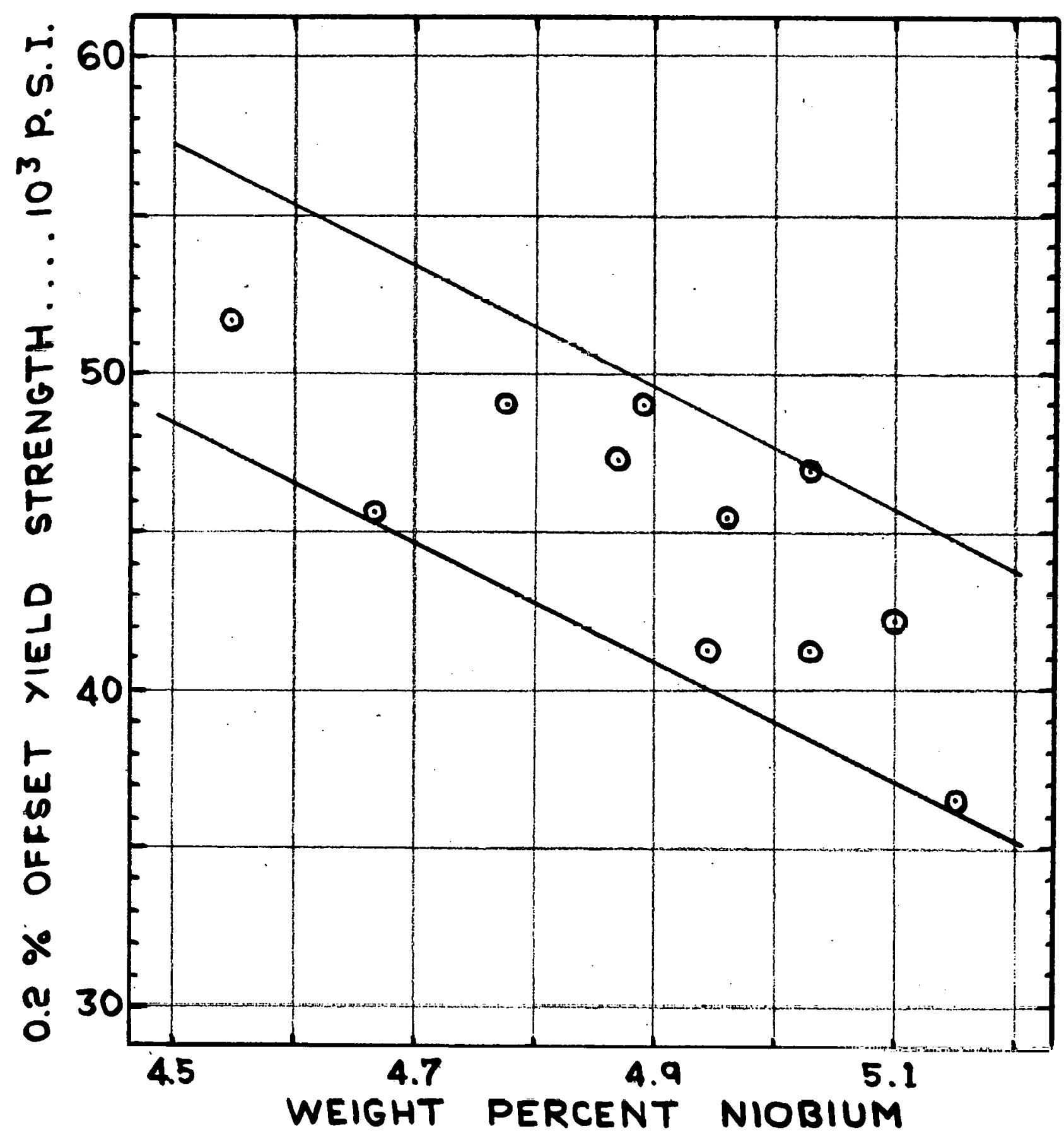

Figure 14. Yield Strength as Function of Composition.

Composition: U-4.5 to U-5.1 wt\% Nb. . . Bar Type: R-3.

Material: Induction cast recycle, homogenized $2 \mathrm{hr} 1100^{\circ} \mathrm{C}$, hot rolled $800^{\circ} \mathrm{C}$, water quenched, tensile blanks machined.

History: Gammaized $30 \mathrm{~min} .800^{\circ} \mathrm{C}$, water quenched, machined, tested $24^{\circ} \mathrm{C}$.

Strain Rate: $\quad 0.005$ in./in.-min. . . Bars per datum point: 1. 


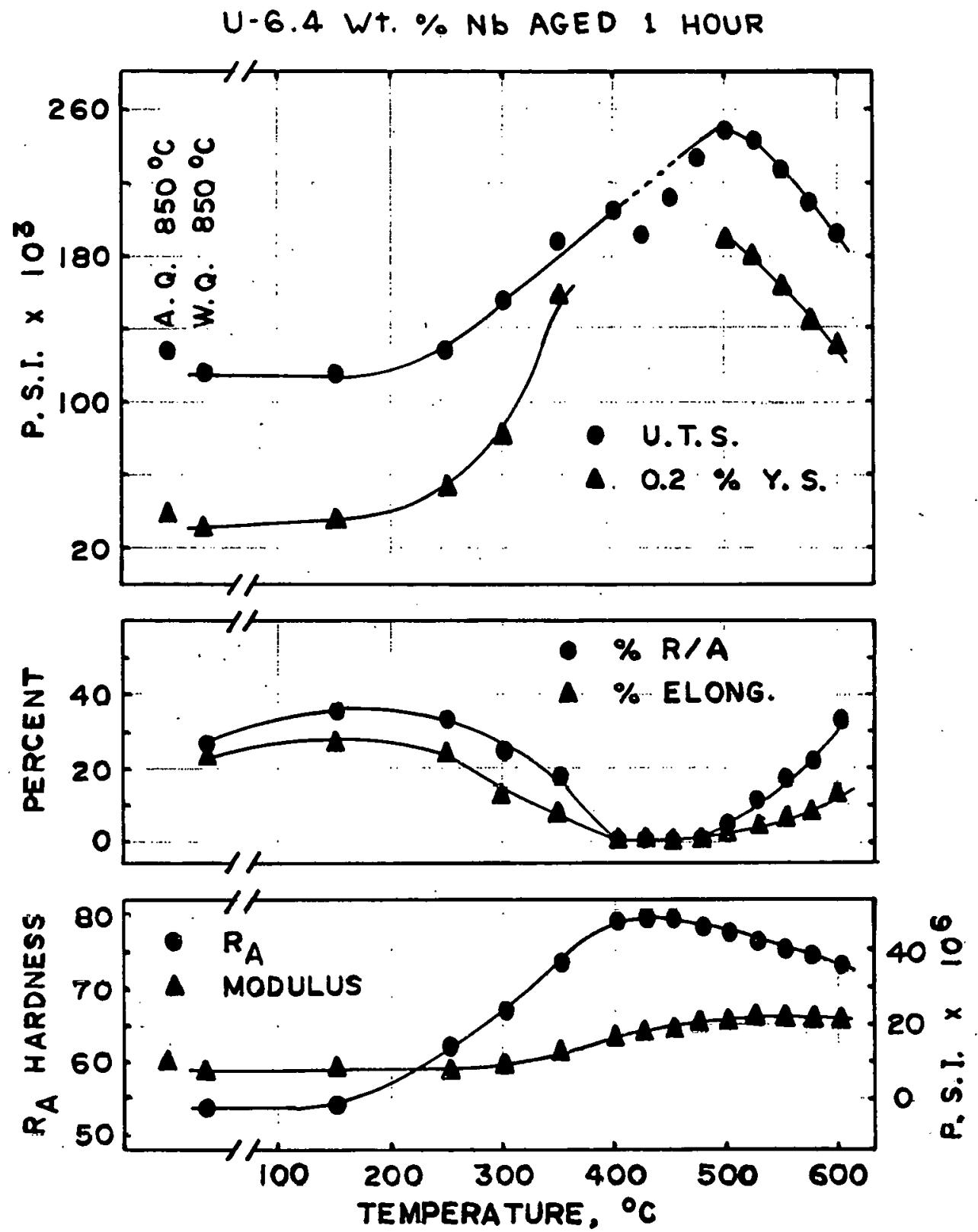

Figure 15. Tensile Test Data for One Hour Aged U-6.3 Nb Alloy.

Composition: U-6.3 wt\% Nb. ... Bar Type: F-1. . . Date 8/66.

Material: Double arc-cast virgin, homogenized $2 \mathrm{hr}$ at $1100^{\circ} \mathrm{C}$, hot rolled $850^{\circ} \mathrm{C}$, water quenched, tensile blanks machined.

History: Gammaized $30 \mathrm{~min} .850^{\circ} \mathrm{C}$, water quenched (6 bars air quenched), aged $1 \mathrm{hr}$ at temperature, air cooled, machined, tesstéd $24^{\circ} \mathrm{C}$.

Strain Rate: $\quad 0.002$ in./in.-min. ... Bars per datum point: 3. 

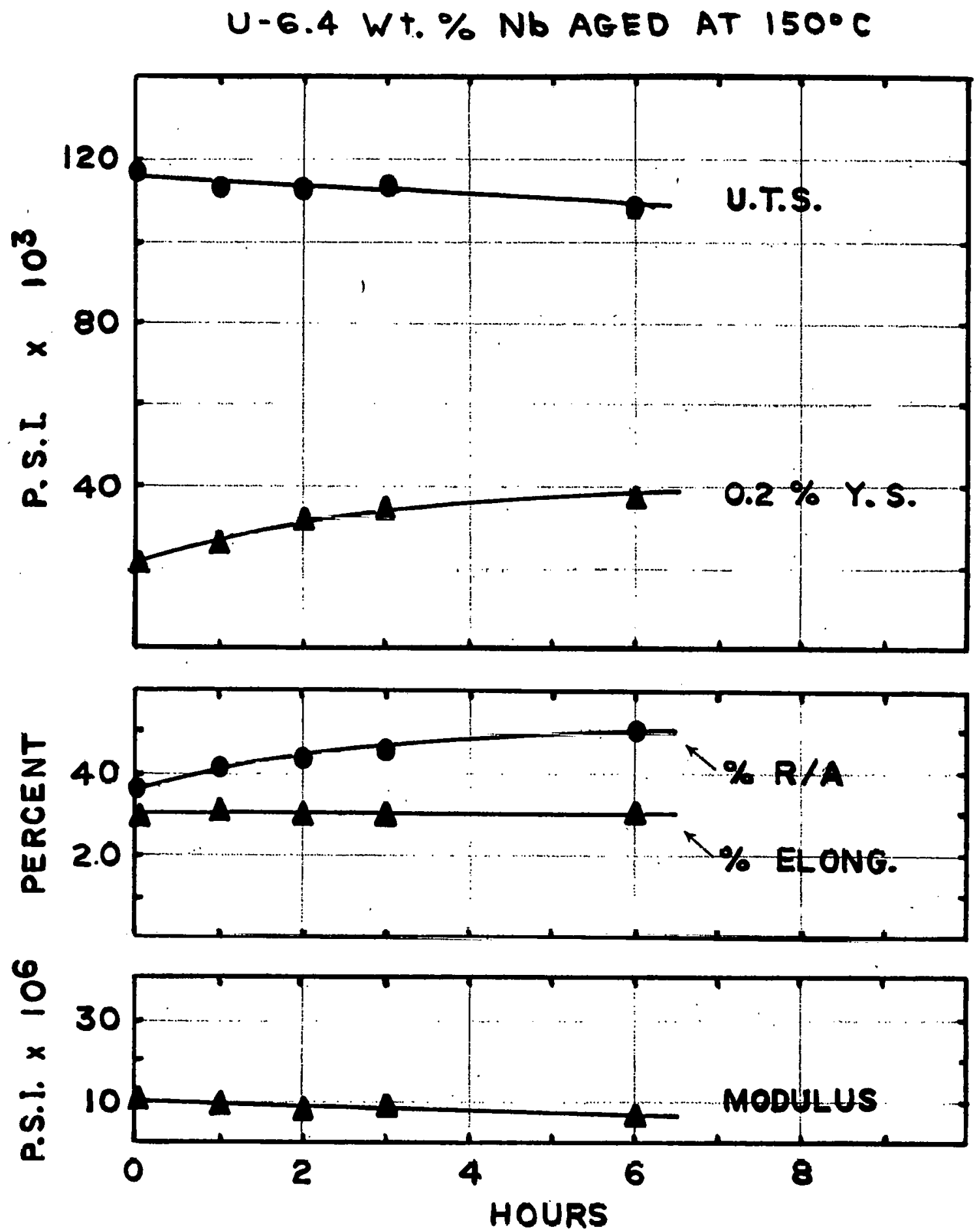

Figure 16. Tensile Test Data for $150^{\circ} \mathrm{C}$ Aged U-6.3 Nb Alloy.

Composition: U-6.3 wt\% Nb. .. Bar Type: F-2. .. Date: $8 / 67$.

Material: Double arc-cast virgin, homogenized $2 \mathrm{hr}$ at $1100^{\circ} \mathrm{C}$, hot rolled $850^{\circ} \mathrm{C}$, water quenched, tensile blanks machined.

History: Gammaized $30 \mathrm{~min}$. at $850^{\circ} \mathrm{C}$, water quenched, aged in oil at $150^{\circ} \mathrm{C}$, air cooled, tested $24^{\circ} \mathrm{C}$.

Strain Rate: $\quad 0.002$ in./in.-min. ... Bars per datum point: 3. 


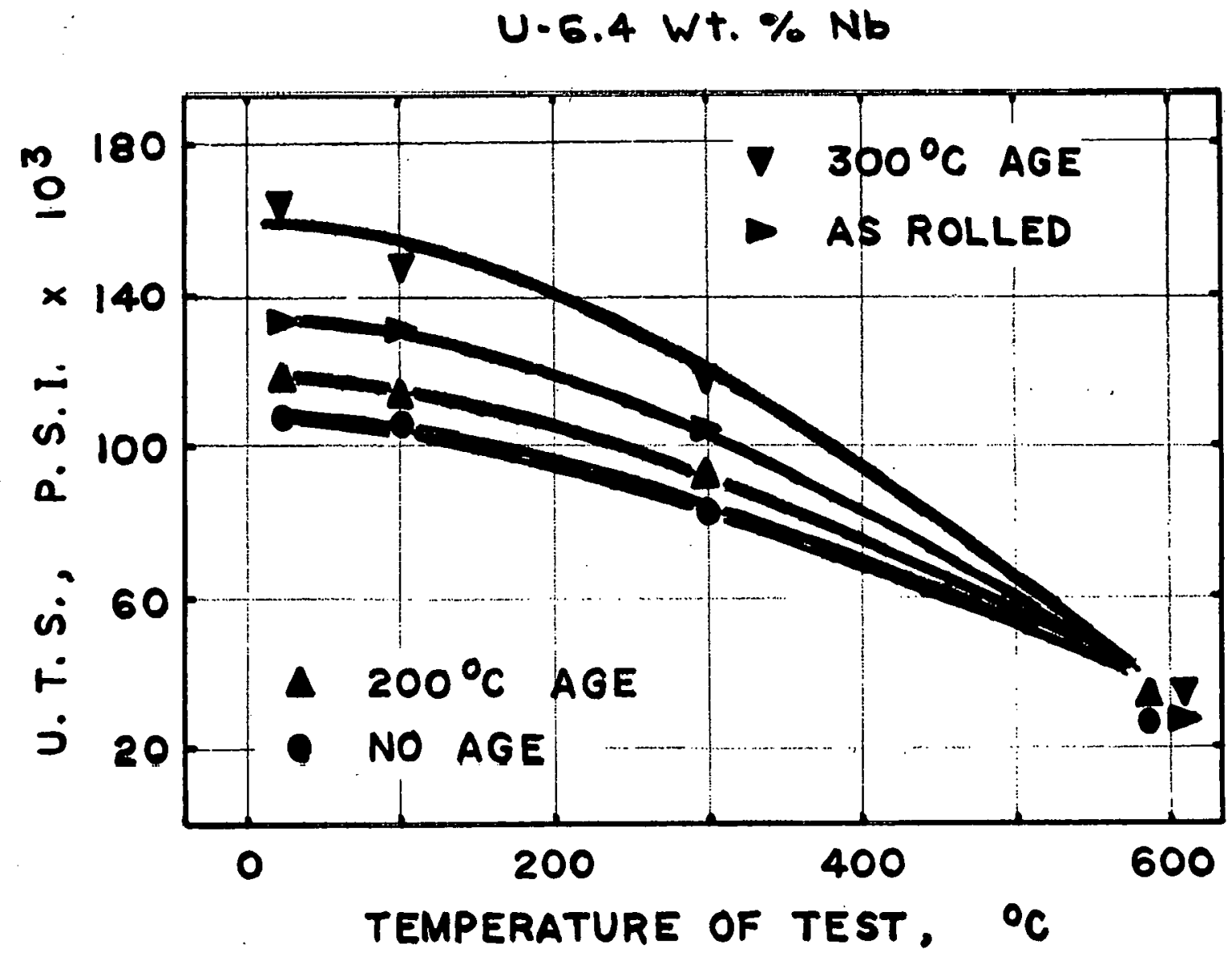

Figure 17. Hot Tensile Test Data for U-6.3 Nb Alloy.

Composition: U-6.3 wt\% Nb. ... Bar Type: F-1... Date: $9 / 65$.

Material: Double arc-cast virgin, homogenized $2 \mathrm{hr}$ at $1100^{\circ} \mathrm{C}$, hot rolled $850^{\circ} \mathrm{C}-550^{\circ} \mathrm{C}$, water quenched, tensile blanks machined, (one set of bars were tested in the as-rolled condition).

Hirtory: Gammaized $30 \mathrm{~min}$. at $850^{\circ} \mathrm{C}$, wator quonehed, aged $2 \mathrm{hr}$ at indioatod temporatura, air coolod, machincd, tostod hot at 100,300 , or $600^{\circ} \mathrm{C}$.

Strain Rate: $\quad 0.002$ in./in.-min. ... Bars per datum point: 1. 


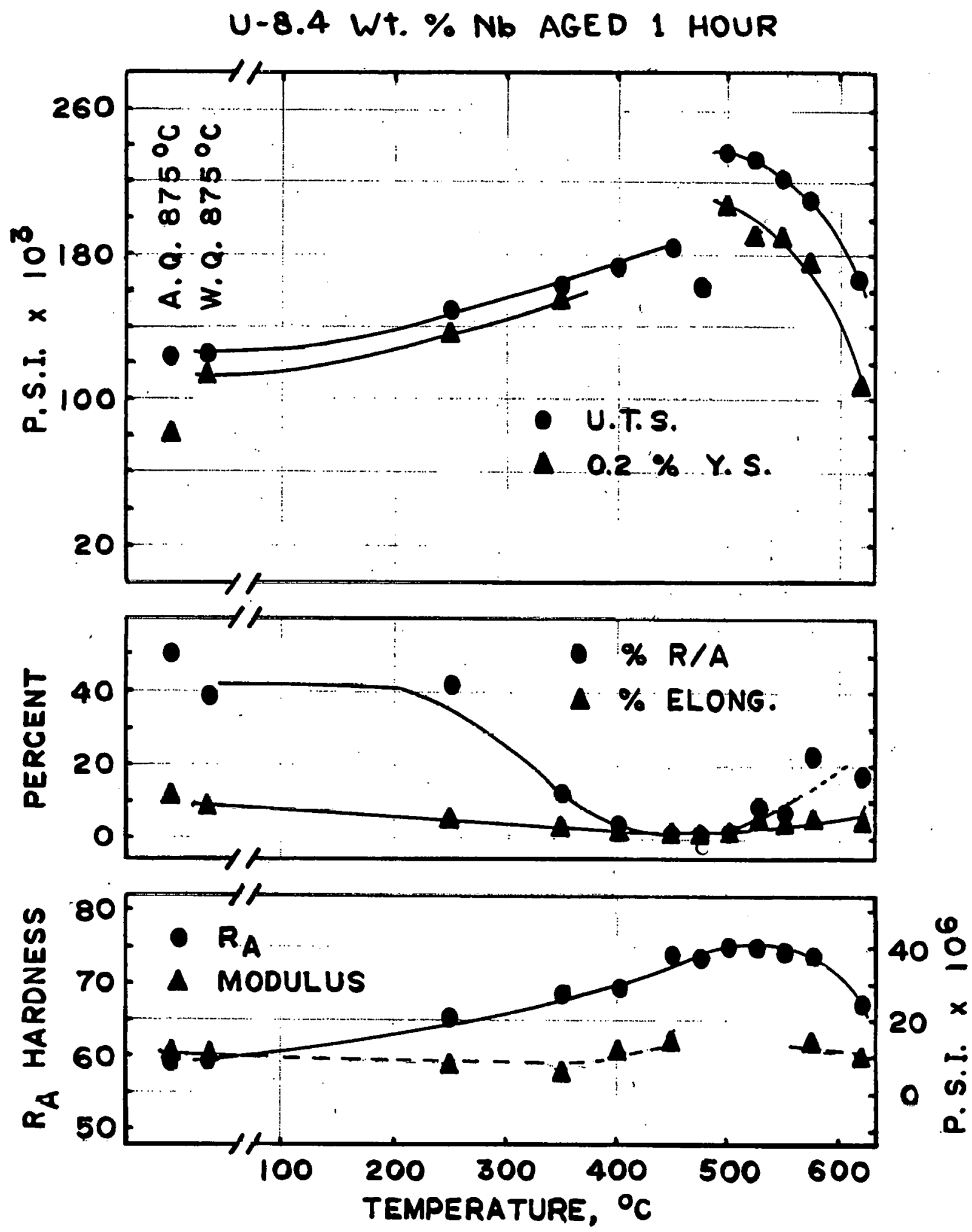

Figure 18. Tensile Test Data for U-8.5 Nb Aged One Hour.

Composition: U-8.4 wt\% Nb. . . Bar Type: F-1... . Date: $1 / 67$.

Material: Double arc-cast virgin, homogenized $2 \mathrm{hr}$ at $1100^{\circ} \mathrm{C}$, hot rolled $850^{\circ} \mathrm{C}$, water quenched, tensile blanks machined.

History: Gammaized $30 \mathrm{~min}$. at $875^{\circ} \mathrm{C}$, water quenched ( 6 bars air quenched), aged $1 \mathrm{hr}$ at temperature, air cooled, machined, tested $24^{\circ} \mathrm{C}$.

Strain Rate: $\quad 0.002$ in./in.-min. ... Bars per datum point: 3 . 
RFP-1703
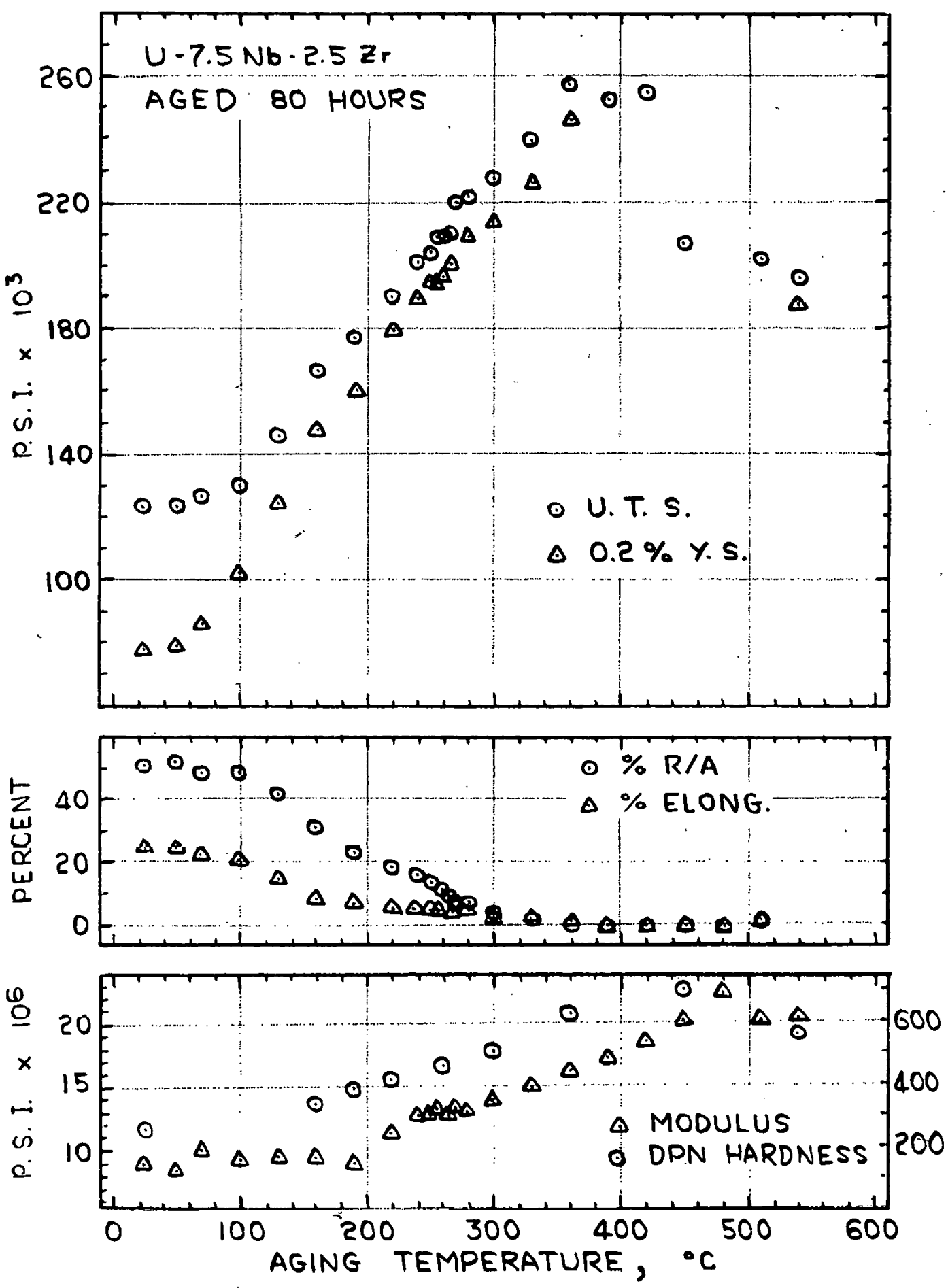

Figure 19. Tensile Test Data for 80 Hour Aged U-7.3 Nb-2.5 Zr Alloy.

Composition: U-7.3 wt\% Nb-2.5 wt\% Zr. . . Bar Type: R-3. . . Date: 3/70.

Material: Inductinn cast, homogenized $4 \mathrm{hr}$ at $1150^{\circ} \mathrm{C}$, hot forged $800^{\circ} \mathrm{C}$, water quonohod, tonsile blenks machined.

Histury: Gammalzed $30 \mathrm{~min}$. at $900^{\circ} \mathrm{C}$, water quèñched, aged $80 \mathrm{hr}$ at temperature, air cooled, machined, tested $24^{\circ} \mathrm{C}$.

Strain Rate: $\quad 0.005$ in./in.-min. . . Bars per datum point: 1 . 

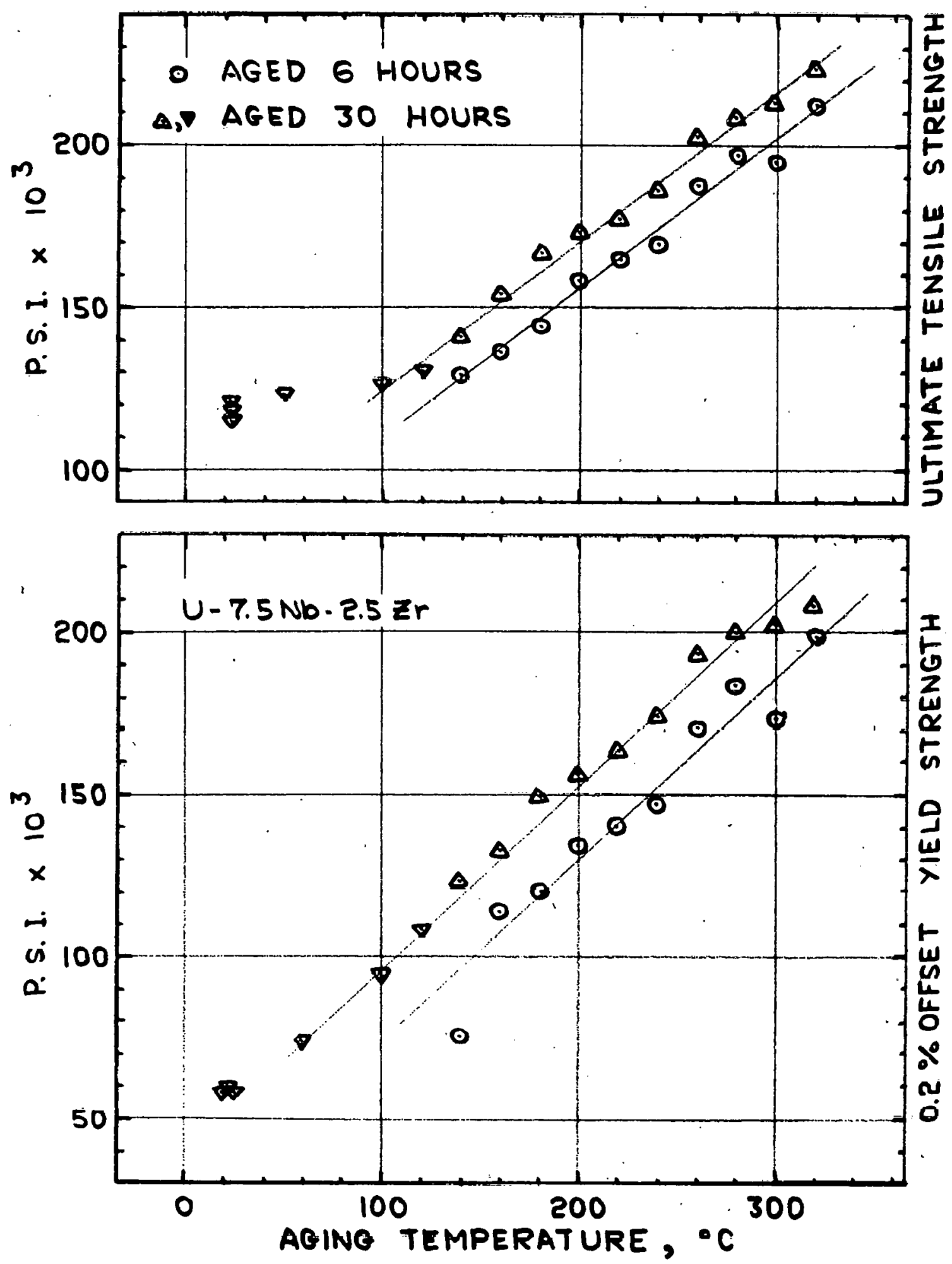

Figure 20a. Tensile Test Data for 6 and 30 Hour Aged U-7.3 Nb-2.5 Zr Alloy.

Composition: U-7.3 wt\% Nb-2.5 wt\% Zr. . . Bar Type: R-3. . . Date: 6/68; $1 / 69$

Material: Induction cast, homogenized $4 \mathrm{hr}$ at $1150^{\circ} \mathrm{C}$, hot forged $800^{\circ} \mathrm{C}$, water quenched, tensile blanks machined.

History: Gammaized $30 \mathrm{~min}$. at $900^{\circ} \mathrm{C}$, water quenched, aged 6 or $30 \mathrm{hr}$ at temperature, air cooled, machined, tested $24^{\circ} \mathrm{C}$.

Strain Rate: $\quad 0.005$ in./in.-min. . . Bars per datum point: 1 for $6 \mathrm{hr}, 2$ for $30 \mathrm{hr}$. 
RFP-1703
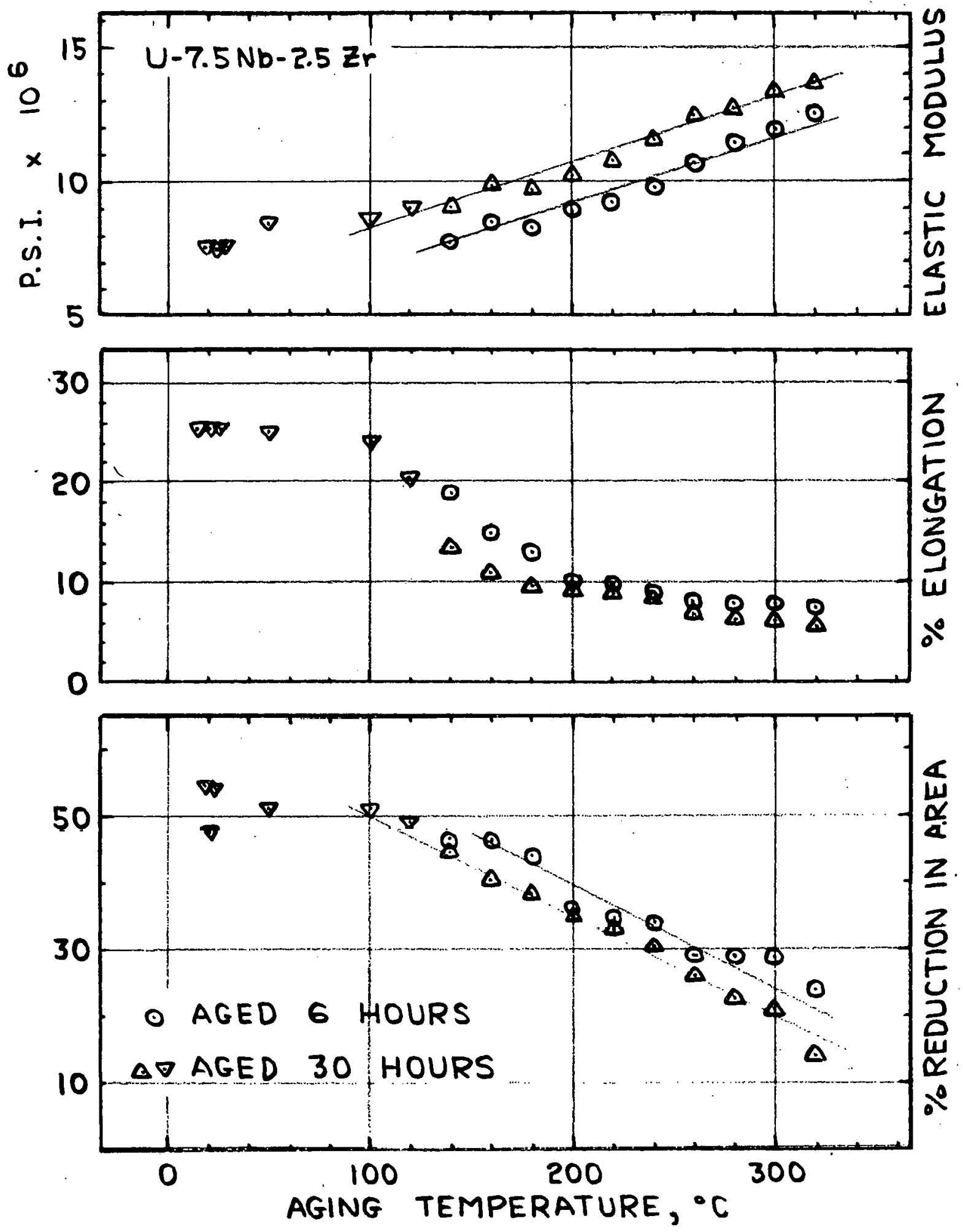

Figure 20b. Tensile Test Data for 6 and 30 Hour Aged U-7.3 Nb-2.5 $\mathrm{Zr}$ Alloy.

Composition: U-7.3 wt\% Nb-2.5 wt\% $\mathrm{Zr}$. . . Bar Type: R-3. . . Date: 6/68; $1 / 69$.

Material: Induction cast, homogenized $4 \mathrm{hr}$ at $1150^{\circ} \mathrm{C}$, hot forged $800^{\circ} \mathrm{C}$, water quenched, tensile blanks machined.

Histnry. Gammaized $30 \mathrm{~min}$. at $900^{\circ} \mathrm{C}$, wator quonohod, aged 6 or $30 \mathrm{hr}$ at tempcrature, air eooled, machined, tested $24^{\circ} \mathrm{C}$.

Strain Rate: $\quad 0.005$ in./in.-min. ... Bars per datum point: 1 for $6 \mathrm{hr}, 2$ for $30 \mathrm{hr}$. 

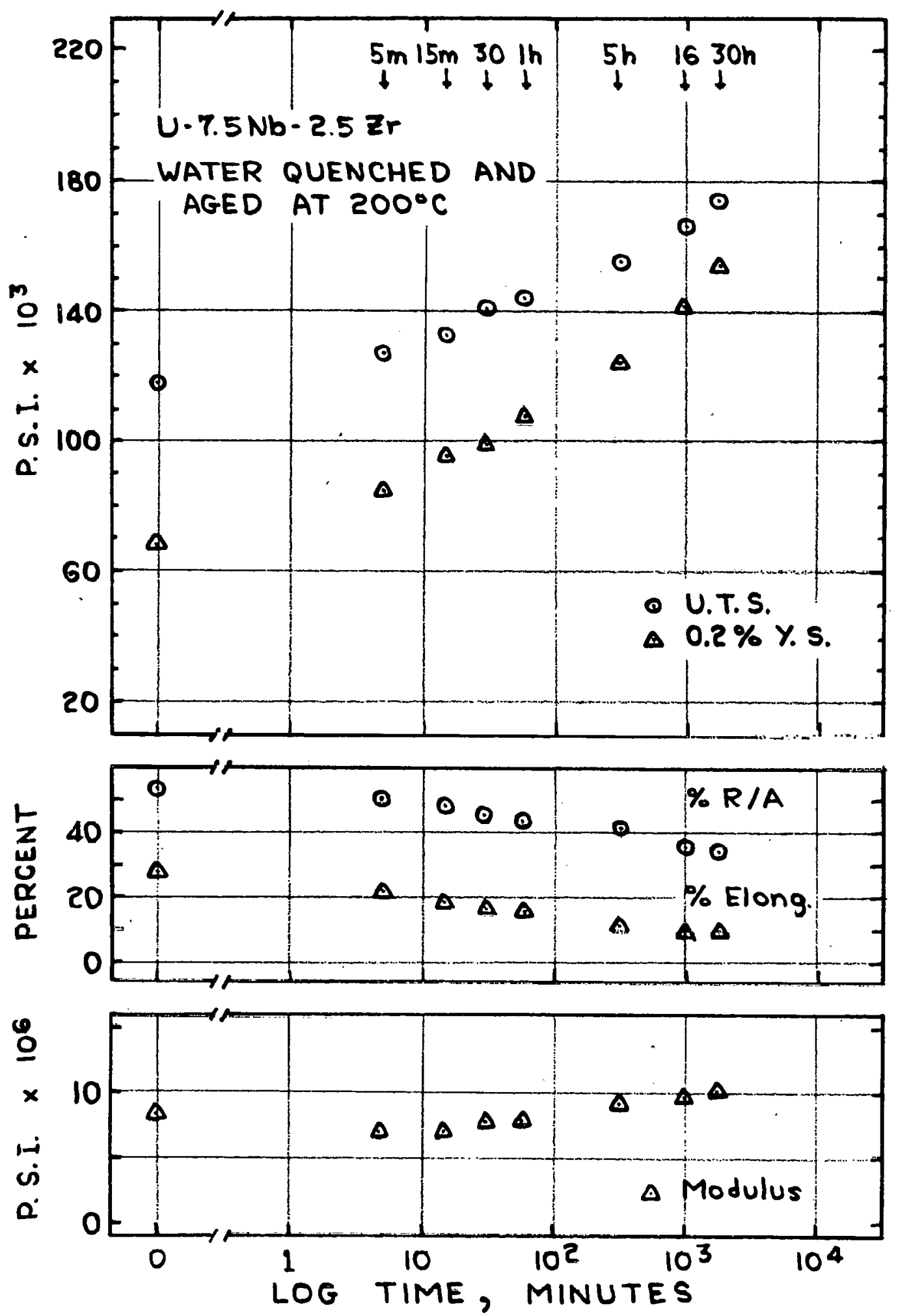

Figure 21. Tensile Test Data for $200^{\circ} \mathrm{C}$ Aged U-7.3 Nb-2.5 Zr Alloy.

Composition: U-7.3 wt\% Nb-2.5 wt\% $\mathrm{Z}_{\mathrm{r}}$. . . Bar Type: R-3. . . Date: $1 / 69$.

Material: Induction cast, homogenized $4 \mathrm{hr}$ at $1150^{\circ} \mathrm{C}$, hot forged $800^{\circ} \mathrm{C}$, water quenched, tensile blanks machined.

History: Gammaized $30 \mathrm{~min}$. at $900^{\circ} \mathrm{C}$, water quenched, aged $200^{\circ} \mathrm{C}$ in oil, air cooled, machined, tested $24^{\circ} \mathrm{C}$. Strain Rate: 0.003 in./in.-min. . . Bars per datum point: 1 . 


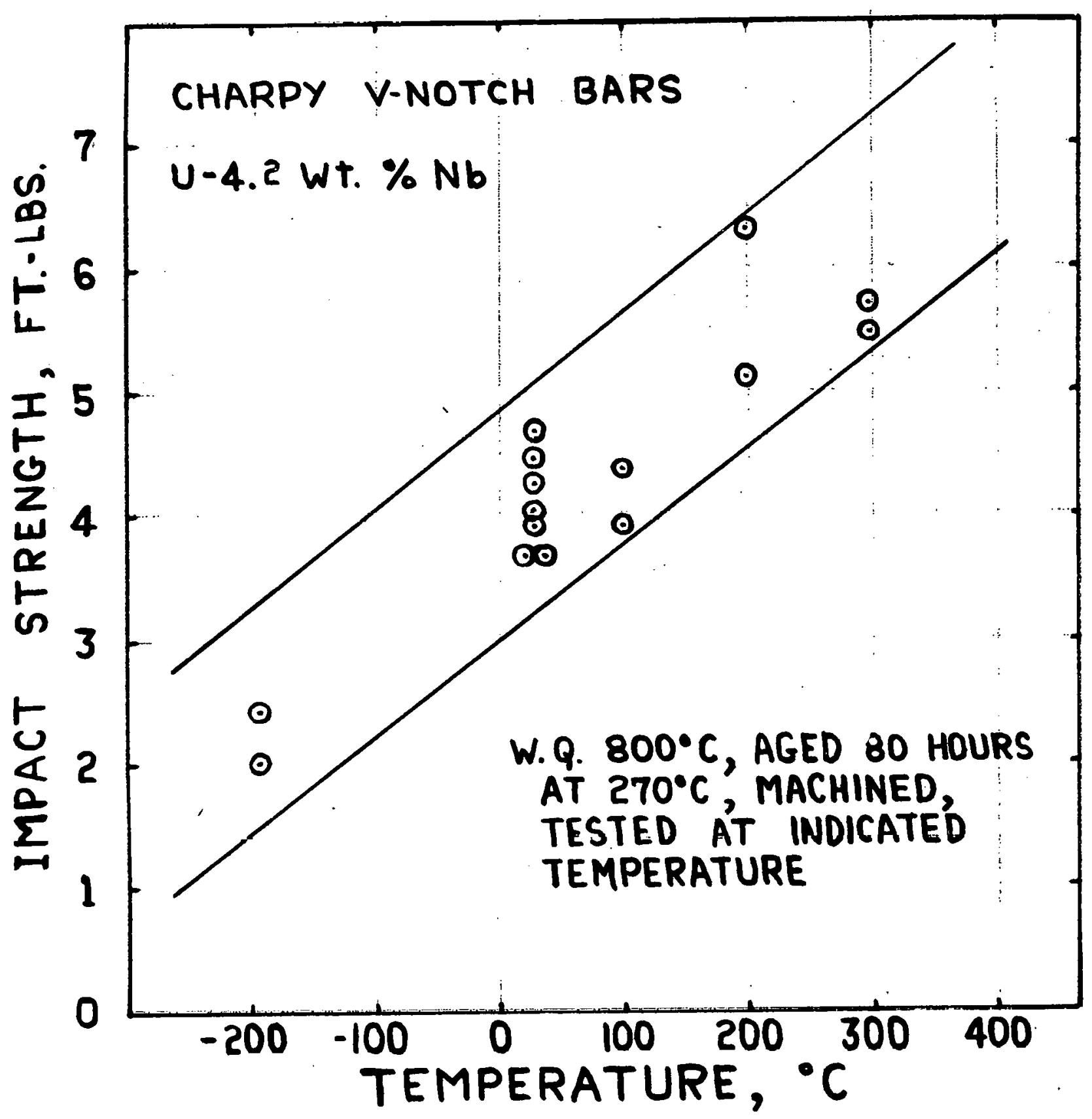

Figure 22. Hot Impact Test Data of 80 Hour Aged U-4.2 Nb Alloy.

Composition: U-4.2 wt\% Nb. . . Bar Type: Std. V-Notch Charpy. . . Date: 10/70.

Material: Induction-cast recycle, homogenized $4 \mathrm{hr}$ at $1100^{\circ} \mathrm{C}$, hot forged $800^{\circ} \mathrm{C}$, water quenched, impact blanks machined.

History. Fammaized $30 \mathrm{~min}$. at $800^{\circ} \mathrm{C}$, uster quenched, aged $80 \mathrm{hr}$ at $370^{\circ} \mathrm{C}$, machined, tectod at indioated tomporaturo. Bars per datum point: 1 . 


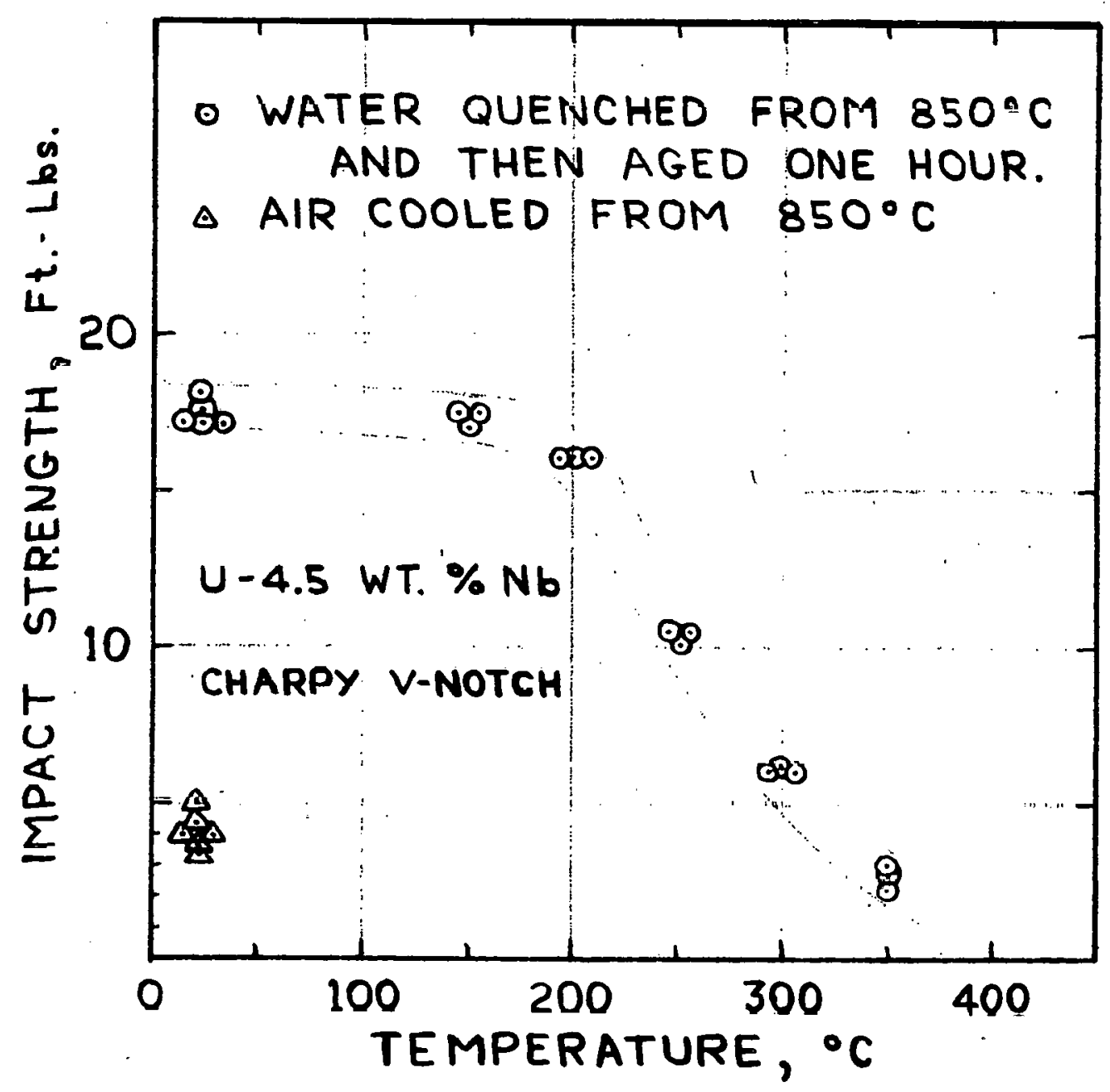

Figure 23. Impact Test Data for One Hour Aged U-4.5 Nb Alloy.

Composition: U-4.5 wt\% Nb.... Bar Type: Std. V-Notch Charpy. . . Date: $1 / 68$.

Material: Induction-cast recycle, homogenized $2 \mathrm{hr}$ at $1100^{\circ} \mathrm{C}$, hot forged $800^{\circ} \mathrm{C}$, water quenched, impact blanks machined.

History: Gammaized $30 \mathrm{~min}$. at $800^{\circ} \mathrm{C}$, water quenched (6 bars air cooled), aged $1 \mathrm{hr}$ at temperature, air cooled, tested $24^{\circ} \mathrm{C}$. Bars per datum point: 1 . 


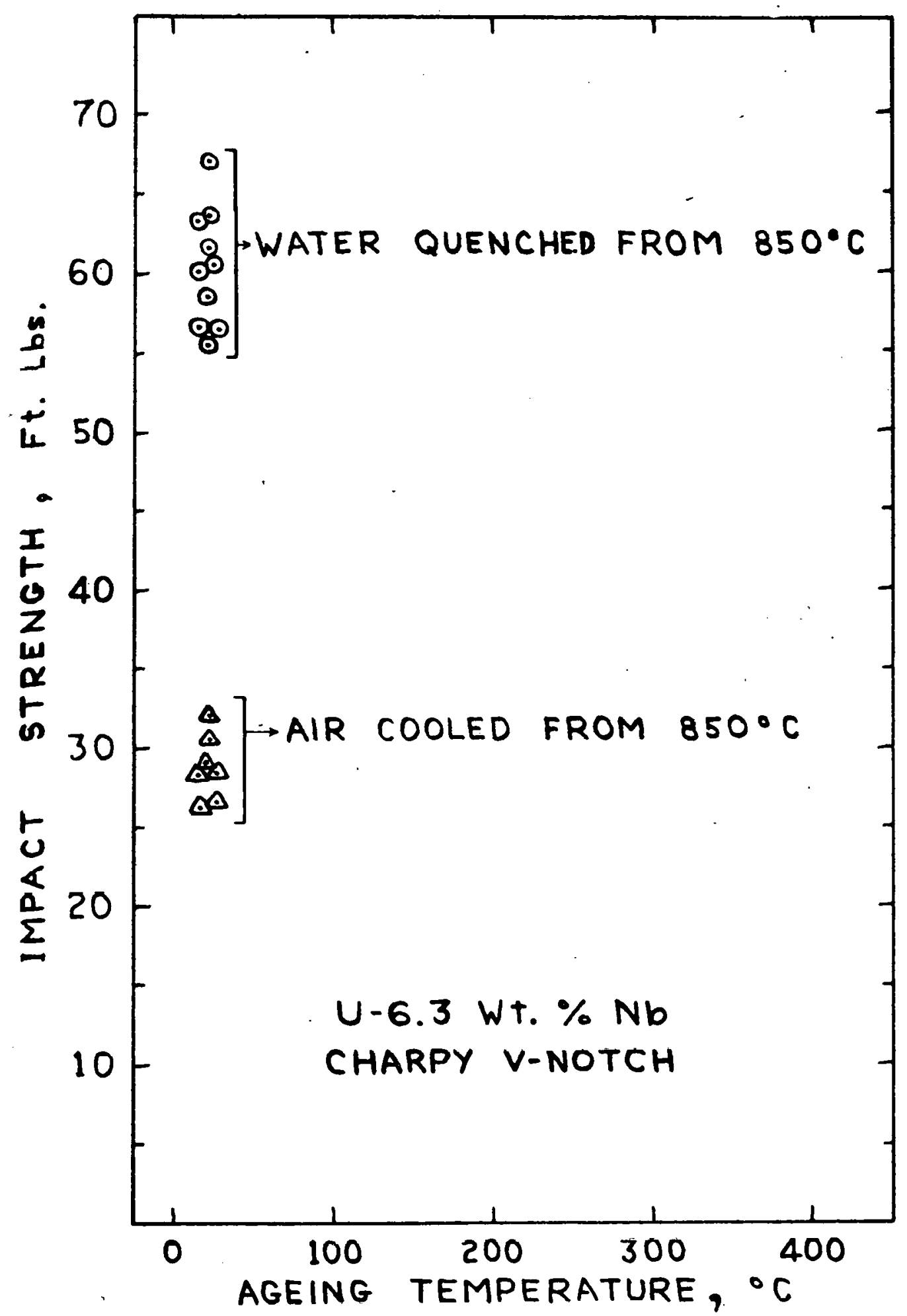

Figure 24. Impact Test Data for U-6.3 Nb Alloy.

Composition: U.6 wt\% Nb. . . Bar Type: Std. V-notch Charpy. . . Date: 9/67, 1/68.

Material: Double arc-cast virgin, homogenized $2 \mathrm{hr}$ at $1100^{\circ} \mathrm{C}$, hot rolled $850^{\circ} \mathrm{C}$, water quenched, impact blanks machined.

History: Gammaizod $30 \mathrm{~min}$. at $850^{\circ} \mathrm{C}$, watcr or air quenclied, maullined, tested $24^{\circ} \mathrm{C}$.

Bars per datum point: 1 . 


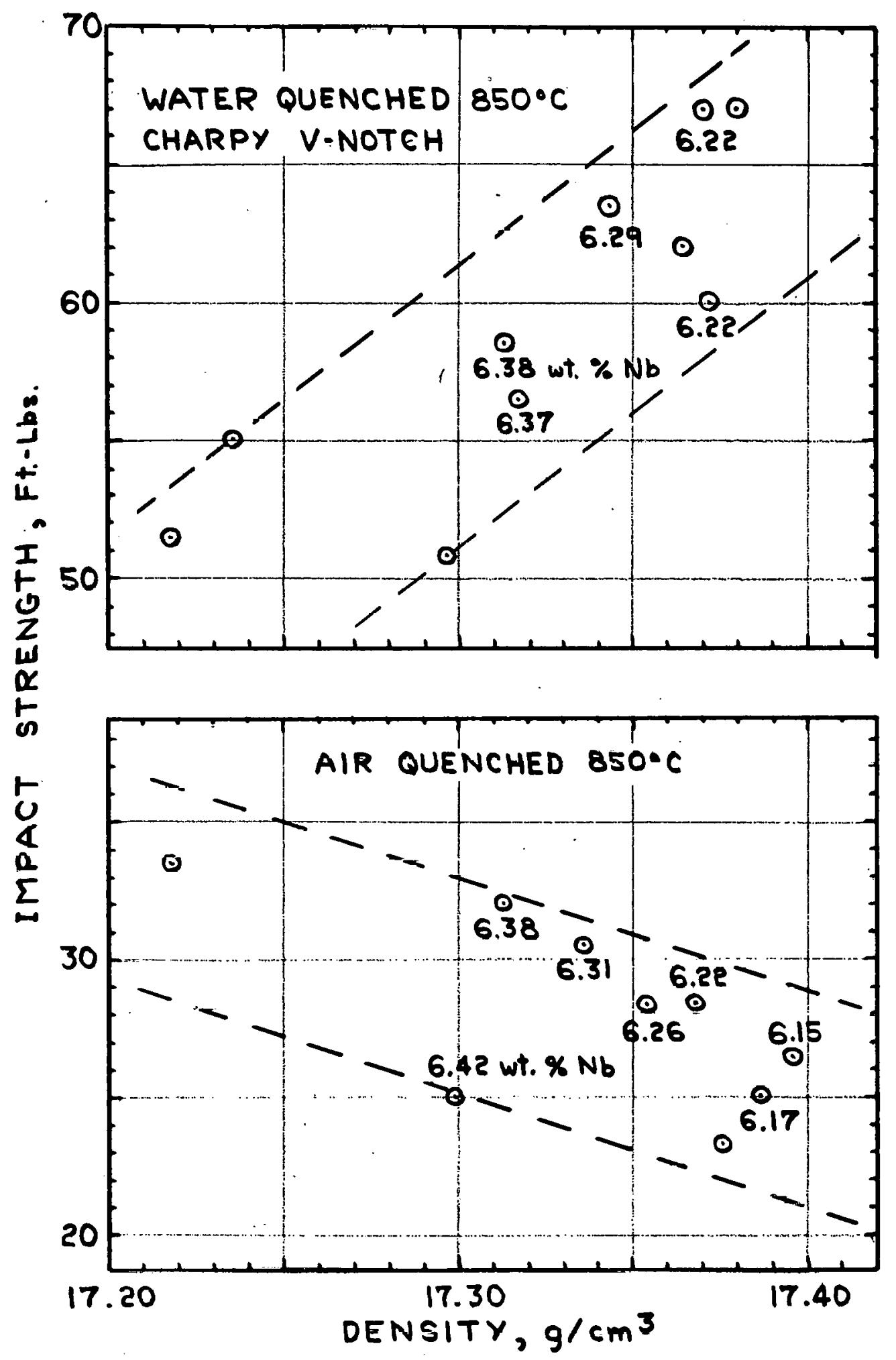

Figure 25. Impact Test Data as Function of Density (Composition). About U-6.3 wt\% Nb. . . Bar Type: V-notch Charpy. . . Date: 9/67, 1/68.

Material: Double arc-cast virgin, homogenized $2 \mathrm{hr}$ at $1100^{\circ} \mathrm{C}$, hot rolled $850^{\circ} \mathrm{C}$, water quenched, impact blanks machined.

History: Gammaized $30 \mathrm{~min}$. at $850^{\circ} \mathrm{C}$, water or air quenched, machined, tested $24^{\circ} \mathrm{C}$. Bars per datum point: 1 . 


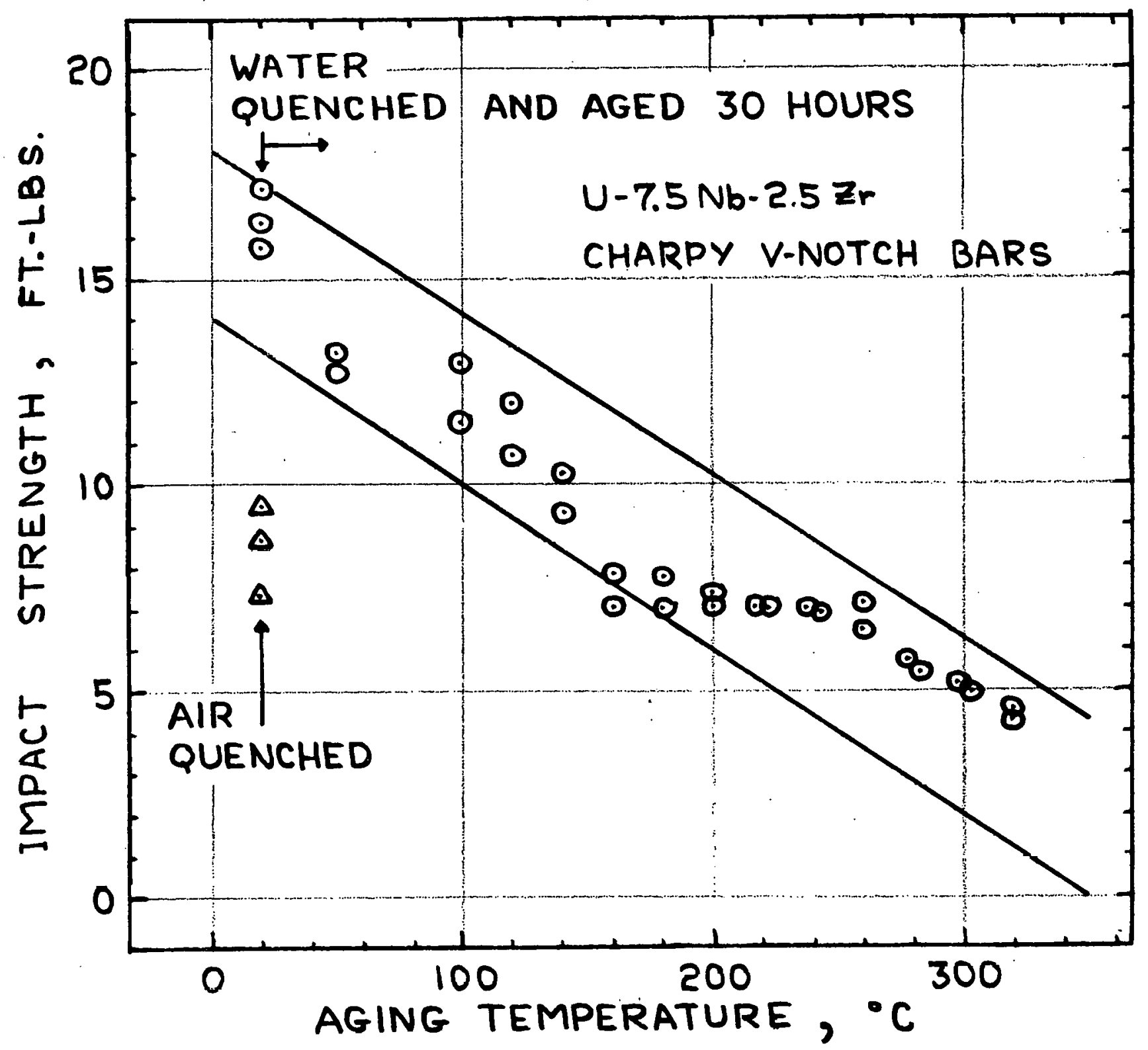

Figure 26. Impact Test Data for 30 Hour Aged U-7.3 Nb-2.5 $Z_{r}$ Alloy.

Compositinn: U-7.3 wt\% Nb-2.5 wt\% Zr... Bar Type: Std. V-notch Charpy. . . Date: $1 / 69$.

Material: Induction cast, homogenized $4 \mathrm{hr}$ at $1150^{\circ} \mathrm{C}$, hot forged $800^{\circ} \mathrm{C}$, water quenched, impact blanks machined.

History: Gammaized $30 \mathrm{~min}$. at $900^{\circ} \mathrm{C}$, water quenched ( 3 air cooled), aged $30 \mathrm{hr}$ at temperature, air cooled, machined, tested $24^{\circ} \mathrm{C}$.

Bars per datum point: 1 . 


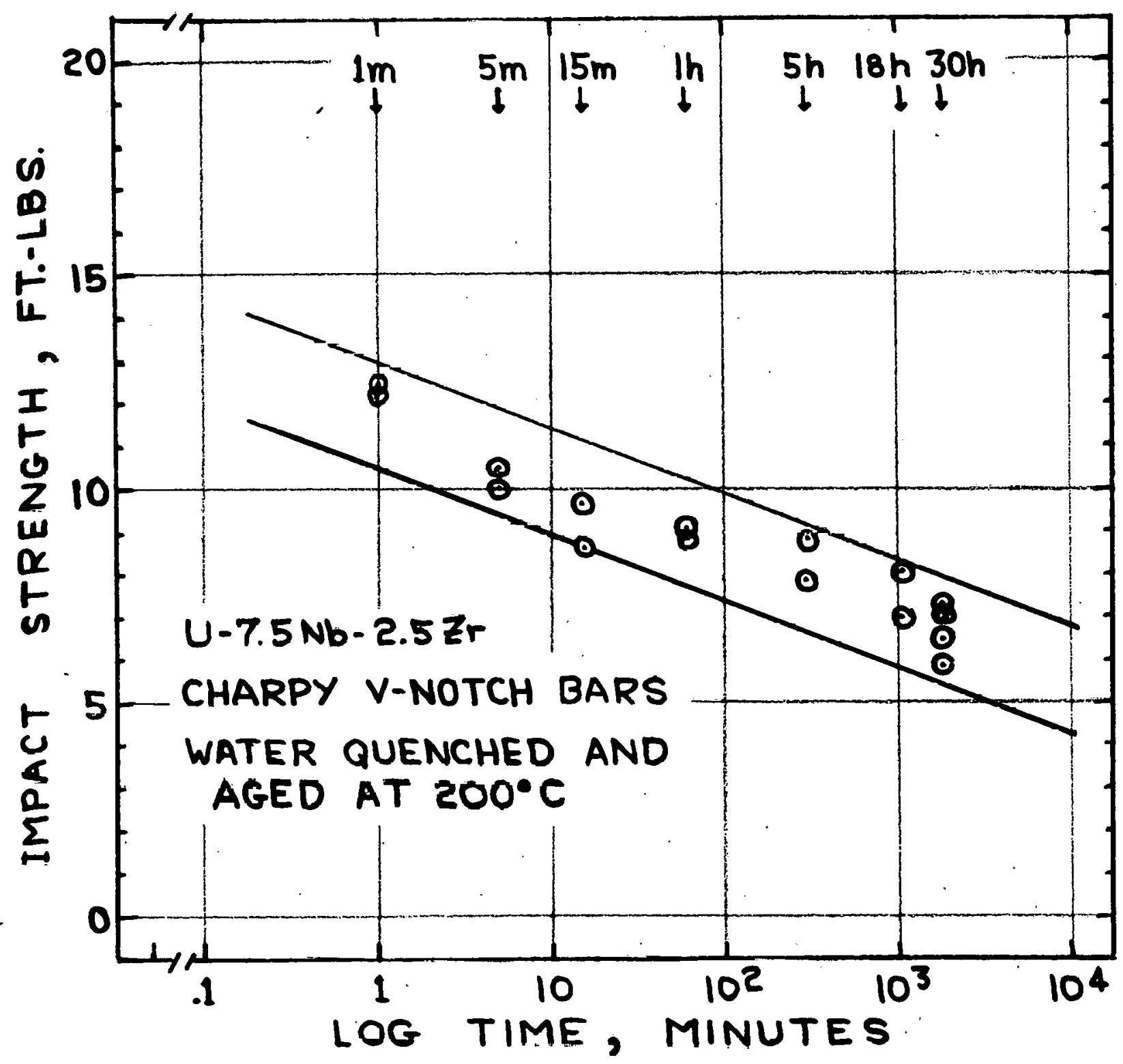

Figure 27. Impact Test Data for $200^{\circ} \mathrm{C}$ Aged U-7.3 Nb-2.5 Zr Alloy.

Composition: U-7.3 wt\% Nb-2.5 wt\% Zr. . . Bar Type: Std. V-notch Charpy. . . Date: 1/69.

Material: Induction cast, homogenized $4 \mathrm{hr}$ at $1150^{\circ} \mathrm{C}$, hot forged $800^{\circ} \mathrm{C}$, water quenched, impact blanks machined.

History: Gammaized $30 \mathrm{~min}$. at $900^{\circ} \mathrm{C}$, water quenched, aged $200^{\circ} \mathrm{C}$ in oil, air cooled, machined, tested $24^{\circ} \mathrm{C}$.

Bars per datum point: 1 . 